Mathématiques et sciences humaines
Mathematics and social sciences

177 | Printemps 2007

Varia

\title{
L'utilisation des modèles structuraux afin d'aborder la complexité du réel en sciences humaines
}

Structural models and complexity of reality

\section{Christian Pellois}

\section{(2) OpenEdition}

\section{Journals}

Édition électronique

URL : http://journals.openedition.org/msh/3817

DOI : $10.4000 / \mathrm{msh} .3817$

ISSN : 1950-6821

Éditeur

Centre d'analyse et de mathématique sociales de l'EHESS

Édition imprimée

Date de publication : 7 mai 2007

Pagination : 53-85

ISSN : 0987-6936

Référence électronique

Christian Pellois, «L'utilisation des modèles structuraux afin d'aborder la complexité du réel en sciences humaines », Mathématiques et sciences humaines [En ligne], 177 | Printemps 2007, mis en ligne le 26 mai 2007, consulté le 23 juillet 2020. URL : http://journals.openedition.org/msh/3817 ; DOI : https://doi.org/10.4000/msh.3817 


\title{
L'UTILISATION DES MODÈLES STRUCTURAUX AFIN D'ABORDER LA COMPLÉXITÉ DU RÉEL EN SCIENCES HUMAINES
}

\author{
Christian PELLOIS ${ }^{1}$
}

\begin{abstract}
RÉSUMÉ - L'analyse statistique implicative élargit le champ du traitement des relations reconnues entre variables. En ce sens elle constitue une avancée importante dans le domaine des modèles structuraux appliqués aux données. Mais, comme tout modèle de traitement, son application en sciences humaines n'est pas sans poser de délicates questions d'interprétation des résultats obtenus. Cet article se propose, à partir de résultats recueillis dans le cadre de recherches antérieures ou en cours, de faire valoir les difficultés méthodologiques que soulève son utilisation.

MOTS-CLÉS - Analyse statistique implicative, Causalité, Degrés de difficulté, Différences, Méthodologie, Précédence

SUMMARY - Structural models and complexity of reality

Implicative statistical analysis expands the field of methods for processing the relationships between variables. In this sense, it represents an important advance in the realm of structural models applied to data. However, as with all processing models, its application in the social sciences poses difficult questions concerning the interpretation of results. Drawing upon the results of both previous and ongoing research, this article aims to evaluate the methodological difficulties of this model.
\end{abstract}

KEY-WORDS - Causality, Degree of difficulty, Differences, Implicative statistical analysis, Methodology, Precedence

\section{INTRODUCTION}

Les modèles mathématiques structuraux appliqués aux données statistiques, en parallèle ou en composition avec d'autres modèles (non linéaires, dynamiques, etc. ${ }^{2}$ ), sont de plus en plus régulièrement utilisés en sciences humaines. Ils permettent de mettre en évidence des structures de co-variations, de régressions, entre différentes variables (analyse multivariée, modèle LISREL), ou de relations implicatives ${ }^{3}$ (logiciel CHIC). Leur utilisation, cependant, n'est pas sans poser des questions, d'ordre méthodologique, délicates à traiter. En effet, les modèles structuraux rendent compte, au regard de la statistique plus classique ${ }^{4}$, de manière plus complète des réalités observées. Mais ils fournissent des résultats dont l'interprétation n'est pas nécessairement aisée à formuler. Par exemple, sous une structure globale observée, quelles sous structures différentielles

1 Laboratoire de psychologie (PPF), UFR de psychologie, Université de Caen, Esplanade de la Paix 14032 Caen cedex, pellois.christian@wanadoo.fr

${ }^{2}$ J. Juhel [2005].

${ }^{3}$ Cf. la définition dans la suite du développement.

${ }^{4}$ Comparaison de moyennes, analyse de variance, etc. 
originales peuvent être dégagées pour des sous populations de la population totale considérée!? Et surtout, quelle compatibilité existe-t-il entre!: la structure ${ }^{5}$ d'ensemble et les structures différentielles correspondant à ces sous populations!?

Ces questions sont quelques-unes des questions qui se sont posées dans le cadre du traitement de données sous modèles structuraux tel que LISREL au plan des co-variations et des calculs de régression [C. Pellois, 2002, 2003], ou tel que le fait valoir le logiciel CHIC sous l'angle des similarités ou de l'analyse implicative. Cet article se propose de présenter, brièvement, des observations faites sous l'angle de l'analyse implicative appliquée au domaine de la psychologie. Seront évoquées, plus particulièrement, dans ce texte, les différentes interprétations possibles auxquelles amènent les résultats obtenus.

Sur quel principe général se fonde l'analyse implicative!? Il convient dès à présent d'en avoir une définition précise. Pour qu'une relation implicative du type «!A implique $\mathrm{B}$ !» soit reconnue il est nécessaire que l'observation d'une valeur de la mesure, correspondant à la performance du sujet, pour une variable donnée A, supérieure à la valeur de la mesure pour une autre variable B, soit inexistante ou suffisamment négligeable, alors que l'inverse peut être couramment observé. L'organisation des données se fait donc selon le cas de figure suivant : présence des couples de valeurs «! $\mathrm{A}+\mathrm{B}+, \mathrm{A}-$ /B-, A-/B+!», et absence ${ }^{6}$, du couple « A+/B- ». La formulation devient, relativement au traitement effectué par le logiciel CHIC!: Une performance réalisée par un sujet à l'épreuve A implique une performance initiale à l'épreuve $B$.

L'article proposé ici est organisé de la manière suivante!:

- un premier développement précise le contexte des observations faites,

- dans un second temps l'ensemble des résultats observés est présenté,

- enfin, des remarques méthodologiques sont soulevées et des analyses interprétatives sont esquissées, à partir de ces «!remarques.

Seront abordées, notamment, les questions relatives!:

- au statut et à la valeur méthodologique du modèle considéré,

- aux utilisations qui peuvent en être faites, dans le cadre de l'analyse scientifique des données, en sciences humaines et, ici, en particulier, dans le domaine de la psychologie.

\section{LE CONTEXTE}

Les travaux, qui ont permis les observations développées dans cet article ont porté, initialement, sur l'influence d'ateliers de remédiation cognitive, au collège, sur les performances et l'éventail des acquis scolaires. Les observations ont été réalisées à partir d'épreuves choisies afin de faire valoir un modèle ouvert du développement psychologique au plan cognitif. Ces travaux ont donné lieu à plusieurs publications antérieures [C. Pellois, 2002, 2003]. Cette publication porte, elle, plus particulièrement, sur la recherche de structures implicatives ${ }^{7}$ sous-jacentes aux performances des élèves observées à la première occasion $^{8}(t 1)$, puis à deux autres occasions $(t 2, t 3)$.

\footnotetext{
${ }^{5}$ De co-variations, de régressions, de similarités, d'implications.

${ }^{6} \mathrm{Ou}$ observé de manière négligeable. Ce qualificatif de «!négligeable!», pour ce type d'analyse statistique, n'a aucun rapport avec la notion «!d'écart négligeable!» tel que défini dans le cadre de l'analyse Bayéso-fiducière (cf. [J. M. Hoc, 1983]).

${ }^{7}$ C. Pellois [2005, en préparation].

${ }^{8}$ Le mot «!occasion!» est pris, ici, dans le sens de mesures faites successivement dans le temps (ici trois
} 
a) Les travaux d'analyse menés, concernant le modèle ouvert du développement psychologique au plan cognitif ont été effectués à l'aide du logiciel d'analyse statistique structurale LISREL. Ils ne seront que succinctement évoqués'.

b) Ceux concernant la recherche de structures implicatives ont été réalisés à l'aide du logiciel d'analyse implicative $\mathrm{CHIC}^{10}$. Ils donneront lieu à une présentation développée tout au long de cet article.

En ce qui concerne l'analyse implicative, un des aspects à élucider, notamment, est de cerner, à l'âge et au niveau scolaire considéré (classes de $6^{\mathrm{e}}$ et de $5^{\mathrm{e}}$ de collège), les performances qui seraient nécessaires, d'un point de vue cognitif, à la maîtrise des contenus scolaires correspondant aux épreuves de contrôle de connaissance de français et de mathématiques. L'hypothèse testée est que la maîtrise de savoirs et savoir faire scolaires implique l'expression de performances au plan des habiletés cognitives ${ }^{11}$ attestées par les scores obtenus par les élèves aux épreuves correspondantes. Par exemple, la capacité à résoudre des problèmes d'arithmétique implique à la fois, des capacités de traitement analogique de données et la maîtrise du raisonnement logique. Mais une autre hypothèse alternative peut être constituée par la proposition inverse!: à savoir l'expression cognitive, son développement, sur contenus variés, nécessite, préalablement, son exercice dans le cadre de disciplines scolaires ${ }^{12}$. Par exemple, la maîtrise du raisonnement logique implique la construction formelle, explicite, et l'entraînement de ce raisonnement dans le cadre des enseignements de mathématiques.

Les épreuves proposées aux élèves sont constituées par les évaluations en français et en mathématiques habituelles à ce niveau d'études et des épreuves psychotechniques qui, pour la clarté de la présentation des résultats retenus ne seront pas toutes évoquées ici. Il sera question dans cet article, seulement, de traiter, au plan cognitif, des performances réalisées par les élèves à «!l'Echelle Collective de Niveau Intellectuel!» (ECNI, cahier IV), et de celles obtenues à une épreuve de «!Mémoire à Court Terme!» (MCT).

Pour rappel, l'évaluation en français, en classe de $6^{\mathrm{e}}$, porte sur quatre domaines!: la compréhension [FR1] ${ }^{13}$, l'expression (traitée dans deux domaines!: [FR2] et [FR3]), la connaissance du code [FR4]. L'évaluation en mathématique porte, elle, sur la maîtrise!: de la numération et des nombres décimaux [M1], des techniques opératoires [M2], du sens des opérations [M3], des figures géométriques, notamment le vocabulaire associé, [M4], des capacités de réception, traitement, production de données mathématiques [M5].

«!L’Echelle Collective de Niveau Intellectuel!» (ECNI) est composée de huit subtests, quatre subtests portent sur le domaine verbal. Il s'agit des épreuves!: 《vocabulaire $»^{14}[\mathrm{VOC}]$, « proverbes $»^{15}[\mathrm{PRO}]$, « analogies verbales $»^{16}[\mathrm{AV}]$, "appartenance à deux classes $»^{17}[\mathrm{~A} 2 \mathrm{C}]$. Les quatre autres portent sur le domaine non verbal, plutôt sur données figuratives ou schématiques. Il s'agit des épreuves!: " séries

\footnotetext{
occasions!: $t 1, t 2, t 3)$, et ceci pour chacune des différentes variables considérées.

${ }^{9}$ Pour une approche plus approfondie, cf. [C. Pellois, 2003(a)].

${ }^{10}$ Cf. [Gras R. et coll., 1996].

${ }^{11}$ Hypothèse "psychologique ».

${ }^{12}$ Hypothèse "pédagogique ».

${ }^{13}$ Les indications entre parenthèses, renvoient aux abréviations utilisées dans les analyses statistiques et reprises ensuite dans le texte.

${ }^{14}$ Maîtrise conceptuelle des mots.

${ }^{15}$ Compréhension verbale.

${ }^{16}$ Faire valoir une règle portant sur le rapport entre les deux premiers termes pour trouver le quatrième terme au regard d'un troisième terme.

${ }^{17}$ Opérateur logique d'intersection de classe sur données lexicales.
} 
numériques $»^{18}[\mathrm{SN}]$, «matrices $»^{19}[\mathrm{MAT}]$, « un élément différent $»^{20}$ [ED], «ifférence $»^{21}[\mathrm{DIF}]$.

L'épreuve de «!'Mémoire à Court Terme ${ }^{22}$ !» porte sur quatre contenus!: des signes schématiques (ponctuation, signes mathématiques, etc.) [Signes], des mots [Mots], des dessins figuratifs schématiques [Images], des chiffres ou nombres à deux chiffres [Chiffres].

Le relevé des scores obtenus à ces différentes épreuves s'est fait dans deux collèges à trois occasions, comme cela a déjà été évoqué ${ }^{23}$. Concernant les mesures à chaque occasion, l'écriture retenue est la suivante!:

- pour les épreuves de contrôle de connaissances en français et mathématiques le numéro correspondant à l'occasion considérée vient après le premier numéro déjà répertorié (cf. ci-dessus), par exemple pour rendre compte du score obtenu en français dans le domaine de la compréhension à la seconde occasion, l'abréviation retenue sera [FR1 2])!;

- pour les épreuves psychotechniques le numéro de l'occasion considérée suit l'abréviation retenue, par exemple, pour le score obtenu à l'épreuve vocabulaire à la première occasion, l'abréviation retenue sera [VOC 1].

Concernant l'ensemble de ces épreuves une autre remarque doit aussi être formulée. Si les épreuves psychotechniques ne présentent aucun inconvénient à ce que leur soit appliqué un traitement quantitatif, les épreuves d'évaluation des connaissances acquises en français et en mathématiques n'ont pas été conçues en ce sens. Même si elles peuvent donner lieu à des résultats quantifiés, l'esprit dans lequel elles ont été conçues est totalement différent. En effet, si ces résultats renvoient à une évaluation sommative et/ou diagnostique, ils réfèrent davantage à un traitement qualitatif dans la perspective de remédiations pédagogiques aux difficultés rencontrées par les élèves dans l'acquisition de connaissances. L'approche quantitative en rapport avec l'évaluation sommative a toutefois été, ici, retenue.

\section{LES RÉSULTATS DE LA RECHERCHE}

\subsection{LE CADRE INITIAL DES OBSERVATIONS ET DES RÉSULTATS RECUEILLIS}

Les résultats obtenus à partir de l'application LISREL, largement développés et commentés par ailleurs [C. Pellois, 2002, 2003], seront ici, seulement évoqués. Ils font

\footnotetext{
${ }^{18}$ Trouver les nombres manquant à une série de nombres après avoir trouvé la (ou les) règle(s) qui les relient.

${ }^{19}$ Cette épreuve est identique à «!analogies verbales!», mais elle porte sur des données figuratives.

${ }^{20}$ Il s'agit de trouver le seul dessin différent de tous les autres.

${ }^{21}$ Il s'agit de trouver, parmi une série de plusieurs figures proposées, la figure la plus différente de la première figure présentée à part (en début de ligne).

${ }^{22}$ Il s'agit, après l'observation pendant 2 minutes de chaque planche, puis le développement d'une autre activité pendant 2 autres minutes, de reproduire, de mémoire, sur une planche vierge, le contenu correspondant à chaque case de la planche initialement observée.

${ }^{23}$ Soit trois moments successifs dans le temps!: au premier trimestre de la scolarité en classe de $6^{\mathrm{e}}$ (octobre, année $n$ ) en fin de classe de $6^{\mathrm{e}}$ (juin, année $n+1$ ), puis en fin de scolarité en classe de $5^{\mathrm{e}}$ (juin, année $n+2)$. Par ailleurs, n'ont été retenus, sur une population totale au départ de 224 élèves, que les élèves présentant la totalité des scores recueillis aux trois occasions, soit 100 élèves.
} 
valoir, au niveau scolaire considéré, que des aides spécifiques, mises en œuvre sous l'angle d'une modélisation particulière, peuvent favoriser le développement cognitif des élèves.

Mais les analyses, y compris statistiques, plus détaillées montrent aussi l'extrême complexité des interactions dans le temps entre les variables considérées et des situations observées lorsque l'on traite ces données de manière différentielle sous plusieurs aspects (classes et ateliers considérés, performances initiales différentielles des élèves, âge, sexe, évolutions individuelles des performances et des profils, etc.).

Par ailleurs le modèle LISREL permet le traitement structural de données en termes de pistes causales, notamment lorsque ces données relèvent, pour les mêmes indicateurs, de prises d'information successives dans le temps, mais il traite de cette question au plan des corrélations ou de la régression multiple et non pas au plan de l'implication tel que peut le faire le logiciel CHIC. Il était donc tentant de vérifier les résultats, obtenus sous LISREL, inférant des influences de variables, les unes par rapport aux autres, inscrites dans le temps, par un outil plus approprié à une recherche de type implicatif tel que le propose le logiciel $\mathrm{CHIC}^{24}$. Les travaux d'analyse «!compréhensive!» développés à partir des résultats obtenus, sur un mode confirmatoire, donneront lieu à une publication ultérieure $^{25}$. Aussi, pour la clarté de l'argumentation, ne seront pris en compte, ici, afin de présenter les éléments d'une réflexion méthodologique concernant les modèles structuraux, que les résultats concernant le second point du traitement abordé ${ }^{26}$ en évoquant seulement quelques éléments en termes d'analyse «!compréhensive!». Il s'agit, en particulier, de faire apparaître non seulement des structures implicatives générales sous jacentes aux performances observées, à une occasion donnée, et pour l'ensemble des occasions successives dans le temps, mais aussi des structures implicatives différentielles en fonction de caractéristiques de sous groupes considérés.

\subsection{LES OBSERVATIONS ATTENDUES}

Elles sont de différents ordres. Tout d'abord, ce qui est recherché ce sont des résultats confirmant les travaux initiaux effectués sous LISREL concernant les effets des ateliers selon une modélisation postulée du développement cognitif. Ces travaux relèvent de la publication spécifique en préparation. Ce qui est ensuite recherché relève des quatre points évoqués ci-dessous.

a) Une structure implicative peut apparaître entre les variables à chaque occasion témoignant d'une possible influence de variables les unes par rapport aux autres.

b) Cette même structure implicative peut être observée à une autre occasion, rendant compte de la permanence dans le temps de la structure des relations des variables considérées entre elles. Ou bien cette structure peut être différente aux différentes occasions, caractérisant ainsi l'évolution dans le temps des relations s'établissant entre les variables considérées.

c) Une structure implicative peut rendre compte des rapports entre les variables dans le temps sous deux aspects. Il s'agit d'une même variable et l'implication rend compte de l'influence de la performance obtenue à une occasion sur la performance obtenue à une

${ }^{24}$ Cf. [R. Gras et coll., 1995!; R. Gras, 1996!; R. Gras et coll., 2005!; M. Bailleul, 1995!; M. Bailleul, 2001] pour une présentation technique!: de l'analyse implicative, de la conception et de l'utilisation du logiciel CHIC.

${ }^{25}$ Notamment, au regard de l'accroissement de performances entre occasions, ce qui n'est pas sans soulever de délicats problèmes méthodologiques de fidélité de la mesure.

${ }^{26} \mathrm{Cf}$. ci-dessus, le point b), p. 55. 
autre occasion. Il s'agit de deux variables différentes et l'implication rend compte de l'influence de la performance obtenue pour une variable à une occasion sur la performance obtenue à l'autre variable à l'occasion suivante.

À priori, il paraît difficile qu'une relation d'implication entre les performances obtenues à deux variables considérées soit différente de l'ordre de successions de ces performances aux différentes occasions.

d) Si une structure implicative apparaît suite au traitement des données de manière globale et pour l'ensemble du groupe considéré, les structures implicatives des sous groupes constitués à partir de ce groupe sont susceptibles de rendre compte des sous parties de la structure globale. L'existence de liaisons observables dans les sous groupes, mais non observables au niveau du groupe entier ${ }^{27}$, peut-il constituer une observation logique au regard des résultats, et sous quelles condition ${ }^{28}$ ?? Cette observation est susceptible, en effet, de poser un délicat problème méthodologique d'interprétation des résultats obtenus au niveau du groupe de référence, comme au niveau des sous groupes, au regard des biais possibles, des interactions différentielles contextes/caractéristiques collectives ou mêmes individuelles. Ce qui pose la question de la relativité des structures, et de leur sens, chacune au regard des autres, en fonction des groupes, des situations, etc.

\subsection{LES OBSERVATIONS RÉALISÉES}

À ce sujet il convient de formuler une remarque préalable. Il a été proposé aux élèves considérés, à la première et à la seconde occasion, l'ensemble des exercices des évaluations initiales de français et de mathématiques. Mais, à la $3^{\mathrm{e}}$ occasion, seule une partie commune aux trois évaluations successives, a été retenue, à la passation des épreuves. Par ailleurs l'évaluation de la mémoire à court terme n'a pas été reprise à cette occasion. Aussi le choix qui a été fait ici a consisté à traiter, en ce qui concerne les occasions 1 et 2, l'ensemble des items des évaluations initiales et ne retenir à la troisième occasion que les résultats obtenus à l'échelle collective de niveau intellectuel (ECNI, cahier IV). Ceci étant précisé les résultats obtenus sont les suivants.

i) À la première hypothèse formulée au point précédent, la réponse est oui. Que ce soit à la première ou à la seconde occasion, sur l'ensemble des données disponibles et aux indices de $.75, .70, .65, .60$, une structure implicative apparaît entre des variables (cf. les Graphes 1, 2 et 3 ci-dessous) parmi l'ensemble de celles considérées.

\footnotetext{
${ }^{27}$ Aux mêmes conditions statistiques de prise d'information.

${ }^{28}$ Par exemple!: une observation sur un sous groupe peut-elle être «!affaiblie!» par la position des autres performances dans le groupe «!parent!»!?
} 


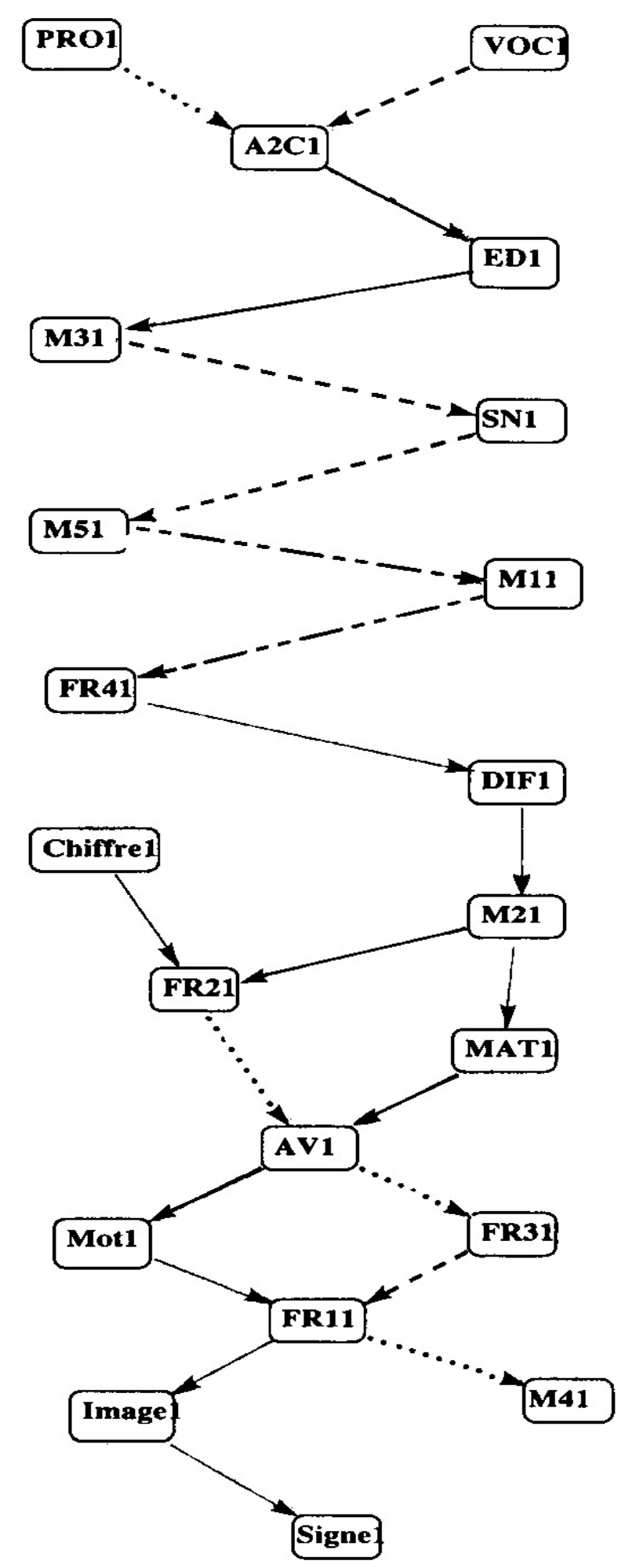

GRAPHE 1. Structure implicative entre les variables à la première occasion Légende. Indices appliqués aux flèches!: 


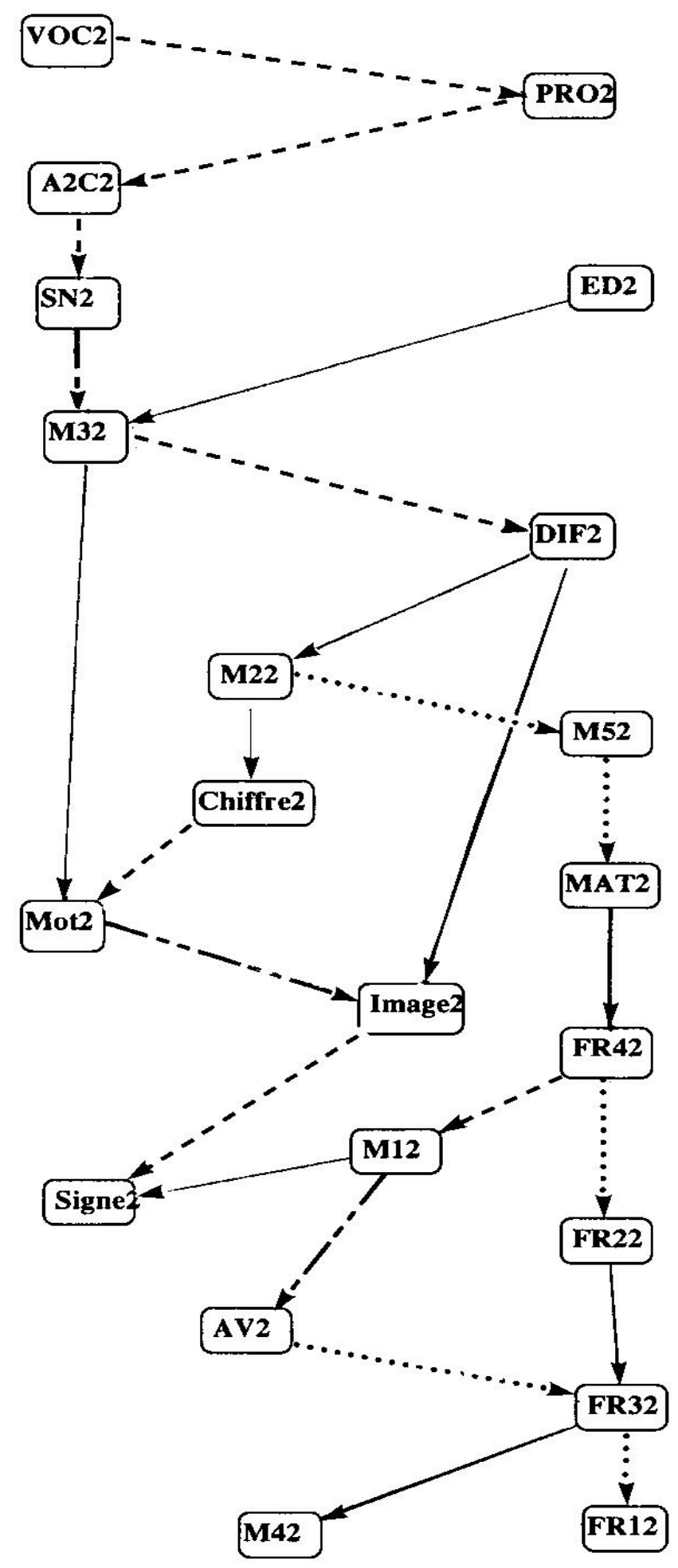

GRAPHE 2. Structure implicative entre les variables à la seconde occasion Légende. Indices appliqués aux flèches!: 


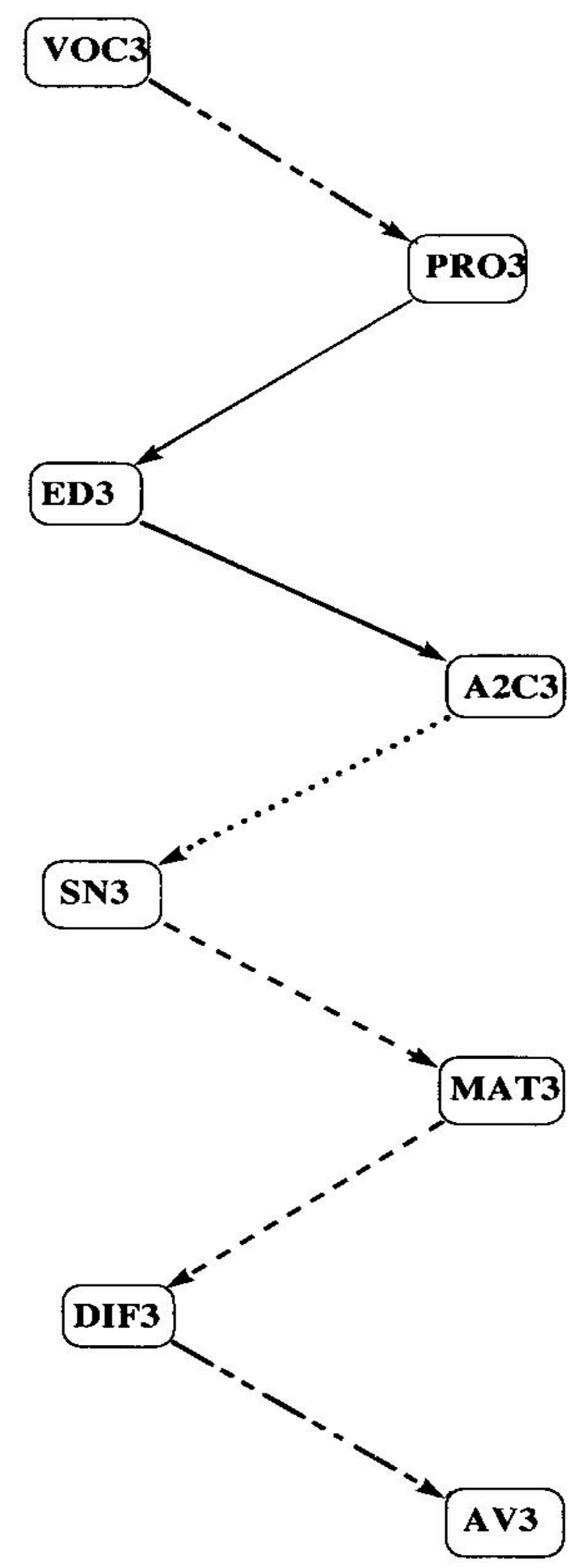

GRAPHE 3. Structure implicative entre les variables observées à partir des mesures partielles effectuées à la troisième occasion

Légende. Indices appliqués aux flèches!: 
Aux seuils choisis, toutes les variables apparaissent dans les structures émergentes, que ce soit à la première ou à la seconde occasion, ou même, pour celles retenues, à la troisième occasion.

ii) En ce qui concerne l'hypothèse alternative, le second point de vue correspond davantage aux résultats obtenus. Des structures différentes entre les variables apparaissent aux deux occasions mobilisant les mêmes variables dans des relations implicatives, identiques entre certaines variables et différentes, d'une occasion à une autre, entre d'autres variables (exemple!: PRO->A2C->ED->M3->SN à l'occasion 1, PRO->A2C$>\mathrm{SN}->\mathrm{M} 3$ à l'occasion 2). Certaines liaisons implicatives apparaissent à une occasion mais pas à une autre et vice versa (exemple!: M2->«Chiffres» à l'occasion 1, VOC$>$ PRO à l'occasion!2; M5->M1->FR4->DIF->M2 à 1'occasion 1, M2->M5 à l'occasion 2).

Pour illustrer le propos, examinons concrètement ce que cela peut signifier en ce qui concerne le premier exemple, proposé ci-dessus. Considérons donc la chaîne implicative développée à la $1^{\text {ère }}$ occasion (PRO->A2C->ED->M3->SN).

SN correspond à l'épreuve!« Séries Numériques ». À partir d'une série de chiffres et de nombres donnée («!2/12/4/9/6/6/8/!»), l'épreuve consiste à trouver, parmi des couples de chiffres et de nombres proposés $(8 / 10,10 / 12,10 / 11,9 / 10,9 / 11,3 ! / 10)$, le couple qui continue la série (à savoir, ici, 3/10). M3 correspond à la partie de l'évaluation $C M 2 / 6^{e}$ en mathématiques portant sur la maîtrise du sens des opérations. Il s'agit, notamment, à la lecture de textes de problèmes nécessitant la réalisation d'une opération, de trouver quel type d'opération arithmétique il convient d'utiliser ceci afin de résoudre le problème posé. En ce qui concerne $\mathrm{ED}$ ( «un Elément Différent»), l'épreuve consiste, à partir d'une bande de 6 dessins géométriques qui se ressemblent d'une certaine manière (cercle, carré, rectangle, losange, etc.), de trouver celui qui est différent (par exemple!: 5 figures sont blanches, une seule est noire, ou bien, 5 dessins sont formés de lignes brisées, un seul est un cercle, etc.). L'épreuve A2C ( Appartenance à 2 Classes ») consiste, à l'intersection de deux séries de mots, une verticale, une horizontale (prénoms de filles/prénoms de garçons, métaux/conjonctions de coordination, départements français/noms de fleuves, etc.), de trouver, dans un ensemble de mots proposés, celui qui convient à l'intersection des deux séries. L'épreuve PRO ( Proverbes ») consiste à trouver parmi 6 phrases («!1. Les souris ne sont pas méfiantes!», «!2. Quand le patron est parti, les apprentis s'amusent!», .. «!6. Les souris aiment danser!»), celle dont le sens se rapproche le plus du proverbe présenté au début de l'exercice considéré («!Quand le chat n'est pas là, les souris dansent!»).

La chaîne implicative indiquerait qu'à cet âge, une performance à un niveau donné, pour une épreuve considérée (par exemple!: un niveau de performances donné à SN "Séries Numériques»), serait «!nécessaire ${ }^{29}$ !» pour qu'apparaisse des performances au plus équivalentes à une autre épreuve (par exemple!: M3 (la maîtrise du sens des opérations dans l'évaluation CM2/6e en mathématiques).

Ce qui est observé à une occasion n'est pas observé à la suivante. Au contraire, nous constatons que cet enchaînement «!implicatif!» (M3->SN) est inversé à la $2^{\mathrm{e}}$ occasion (SN->M3).

iii) Les hypothèses correspondantes au point (c) précédent (cf. p. 57-58) doivent être traitées sous deux aspects à partir du GRAPHE 4, global, qui suit.

${ }^{29} \ll$ !Précèderait!?!» 


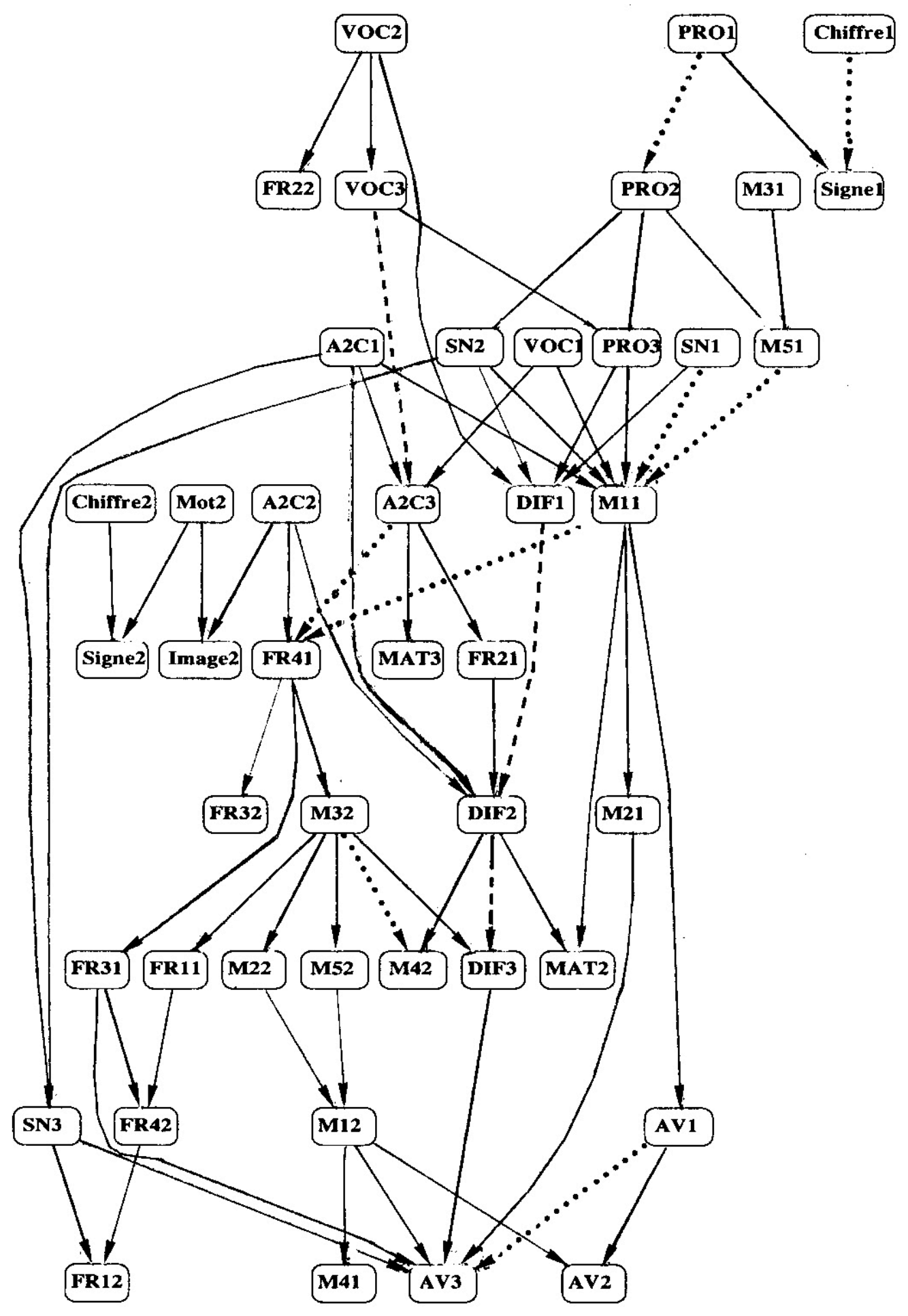

GRAPHE 4. Structure globale des implications entre les performances mesurées à trois occasions

Légende. Indices appliqués aux flèches!:

—.$-: .90 \quad-----: .85$ $: .80$ $: .75$ 
Les indices «!seuil!» choisis, pour rendre compte au mieux des résultats, dans une clarté d'agencement des données satisfaisante, à des seuils paraissant encore suffisants pour rendre compte de l'hypothèse implicative ont été fixés, ici, respectivement, à $.90, .85$, $.80, .75$.

\section{- Premier traitement des résultats obtenus : l'implication entre les différentes mesures} pour une même variable aux trois occasions successives

À ces différents seuils, une variable n'apparaît pas dans la structure implicative, il s'agit de la variable ED. Pour une part des variables, il y a des relations d'implication directe pour les différentes mesures de la même variable aux trois occasions. C'est le cas, par exemple, de PRO et DIF. Pour d'autres, il n'y a pas de relations, mêmes indirectes, entres mesures portant sur la même variable aux trois occasions. Il en est ainsi pour MAT. Une relation peut exister entre deux mesures mais la relation à la troisième n'apparaît pas, de manière cohérente aux deux premières, dans des liaisons à d'autres niveaux de la structure implicative (cf. A2C, SN, VOC, AV, etc.). Une relation implicative entre deux mesures peut exister, mais la troisième mesure n'apparaît même pas dans la suite de la structure. C'est le cas, par exemple, et à nouveau, de la variable MAT. Par contre, il existe beaucoup de relations d'implication entre, au moins, deux mesures se succédant dans le temps (cf. VOC, AV, SN, A2C, PRO, DIF, etc.). Mais toutes ces liaisons implicatives sont le plus souvent inversées au regard de l'hypothèse temporelle initialement formulée. En effet, le réseau implicatif se développe, lorsqu'il existe et plus ou moins directement, de la mesure effectuée à l'occasion 1 vers la mesure effectuée à l'occasion 2, puis vers la mesure effectuée à l'occasion $3^{30}$ et non pas le contraire.

- Second traitement des résultats obtenus : l'implication entre les différentes mesures effectuées pour les différentes variables aux trois occasions

Des observations attendues peuvent être relevées, à savoir!:

- le résultat obtenu pour une variable à une occasion donnée implique le résultat obtenu pour une autre variable à cette même occasion. C'est le cas, par exemple, de A2C 2 qui implique «!Image!» 2 et PRO 2 qui implique SN 2!;

- le résultat obtenu pour une variable à une occasion donnée implique le résultat obtenu pour une autre variable à une occasion précédente. C'est le cas, par exemple, de VOC 2 et SN 2 qui implique DIF 1, de PRO 2 qui implique M5 1 et de PRO 3 qui implique DIF 1 et M1 1 .

D’autres résultats sont plus délicats à interpréter :

- le résultat obtenu pour une variable à une occasion donnée implique le résultat obtenu pour une autre variable à l'occasion suivante. C'est le cas, par exemple, de A2C 1 qui implique DIF 2 ou SN 3, de M1 2 qui implique AV 3, de FR4 1 qui implique M3 2, etc.

iiii) Qu'en est-il des résultats lorsque des structures implicatives sont recherchées dans des sous-groupes de la population totale constitués en fonction de différents critères $^{31}$ !?

\footnotetext{
${ }^{30}$ À savoir 1 implique 2 qui implique 3... (Cf. l'analyse de ces résultats effectués au point 4 . notamment pages 72 et suivantes).

${ }^{31}$ Ici les niveaux de performances sur deux dimensions.
} 
Les choix effectués ont consisté à diviser deux fois la population globale en deux sous groupes, la première fois en fonction de la performance obtenue à la première occasion à l'épreuve «!Matrices!» (MAT), la seconde fois en fonction de la performance obtenue à la première occasion à l'épreuve «!Vocabulaire!» (VOC). La répartition entre les deux groupes, sur chacune des dimensions retenues, s'est faite en tenant compte des performances réalisées, pour chacune des variables, au dessus (MAT+, $\mathrm{VOC}+$ ) ou au dessous (MAT-, VOC-) de la moyenne des performances obtenues par le groupe de référence constitué par l'ensemble de la population des élèves considérés. Le choix de ces sous groupes est conditionné, au regard des dimensions retenues, par un jeu alternatif (vicariance) d'hypothèses psychologiques référent aux buts fonctionnels ${ }^{32}$ attribués au modèle testé ${ }^{33}$. Les résultats obtenus sont présentés dans les quatre Graphes ci-dessous.

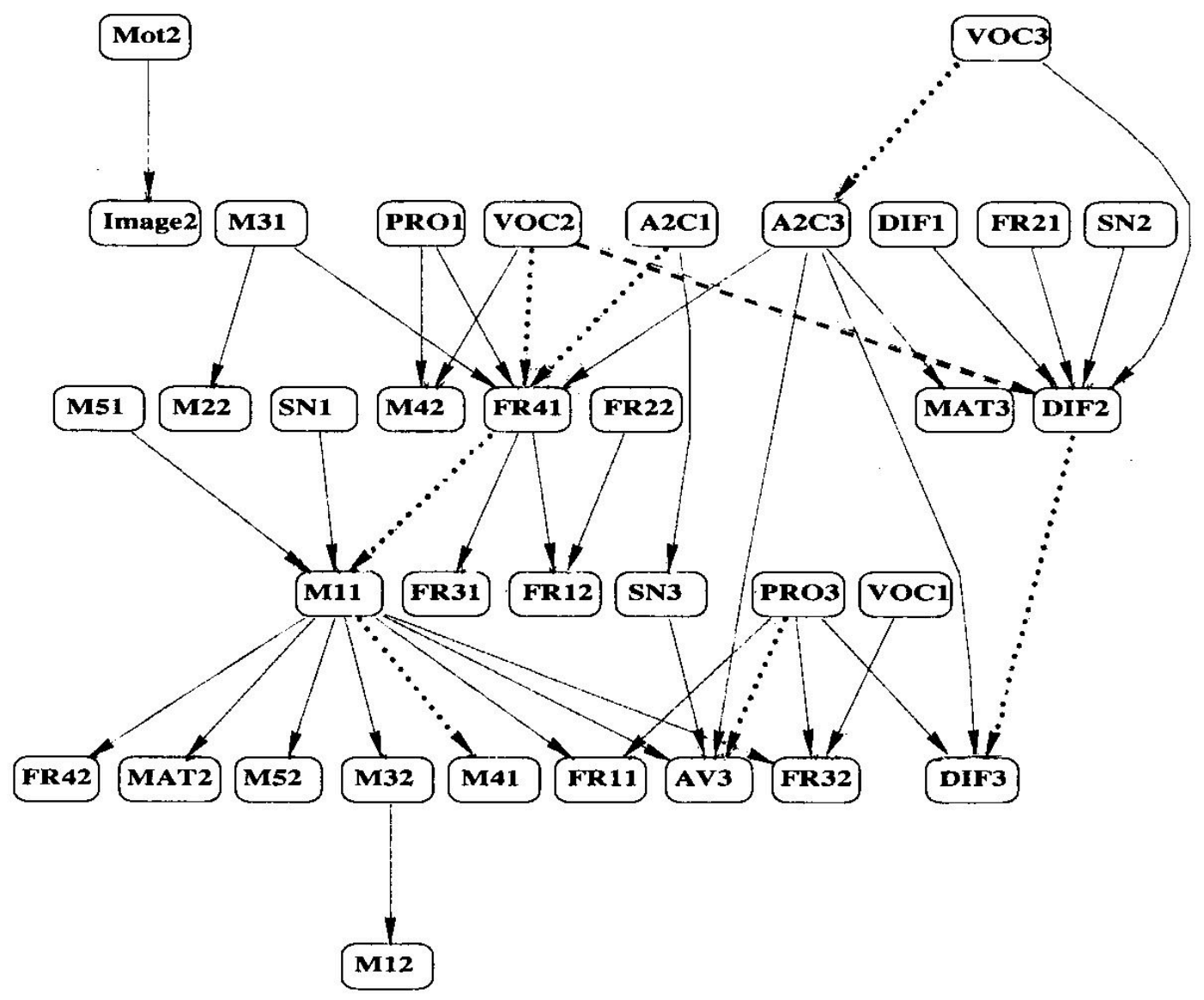

GRAPHE 5. Structure des implications entre les performances mesurées à trois occasions pour les élèves les plus performants à l'épreuve «!Matrices!»

Légende. Indices appliqués aux flèches!:

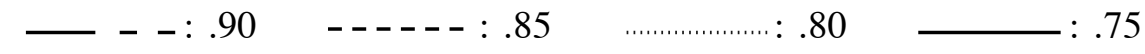

${ }^{32}$ Cf. [Pellois, 2002, 2003], déjà cité.

${ }^{33} \mathrm{Cf}$. début du point 2. 


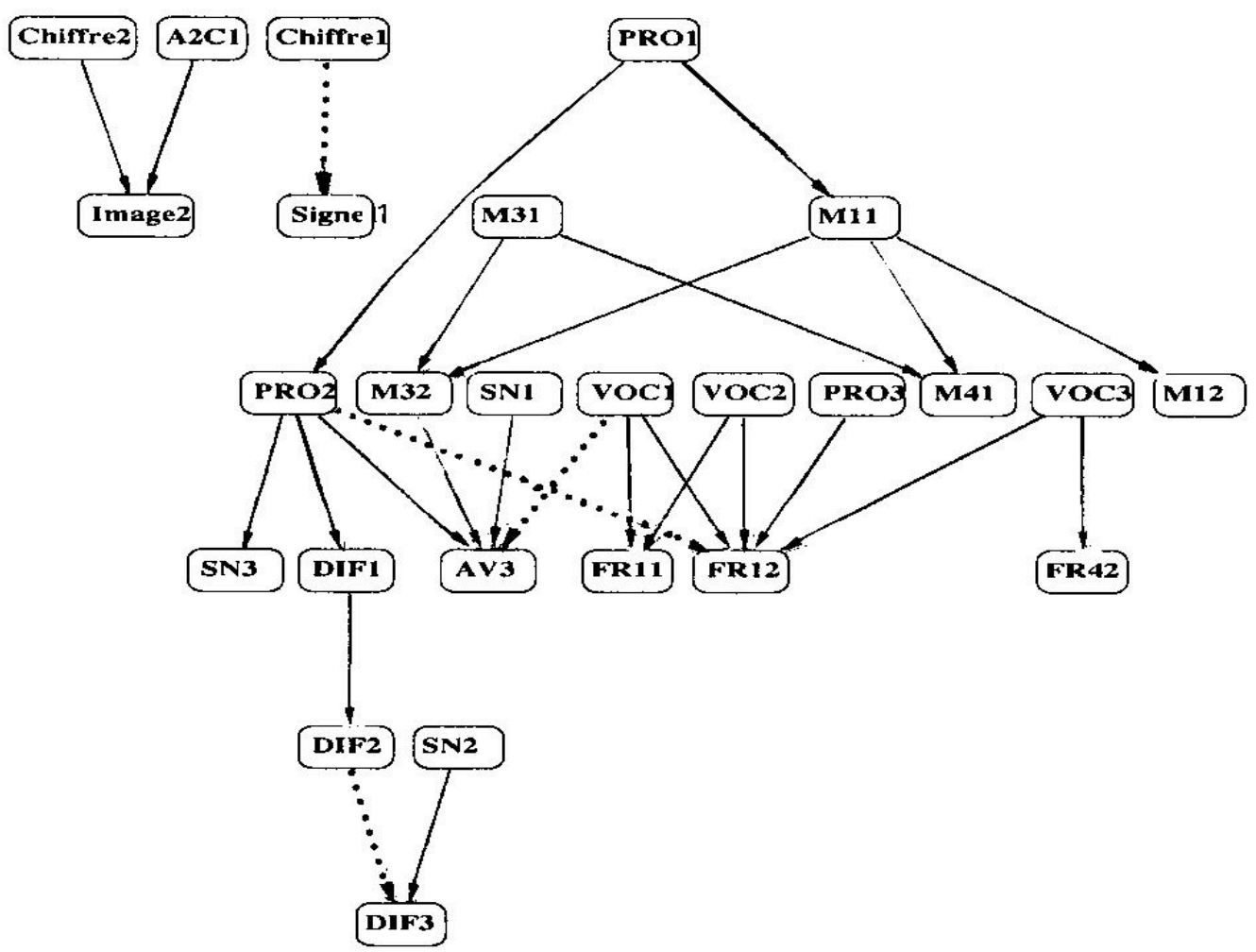

GRAPHE 6. Structure globale des implications entre les performances mesurées à trois occasions pour les élèves les moins performants à l'épreuve «!Matrices!»

Légende. Indices appliqués aux flèches!:
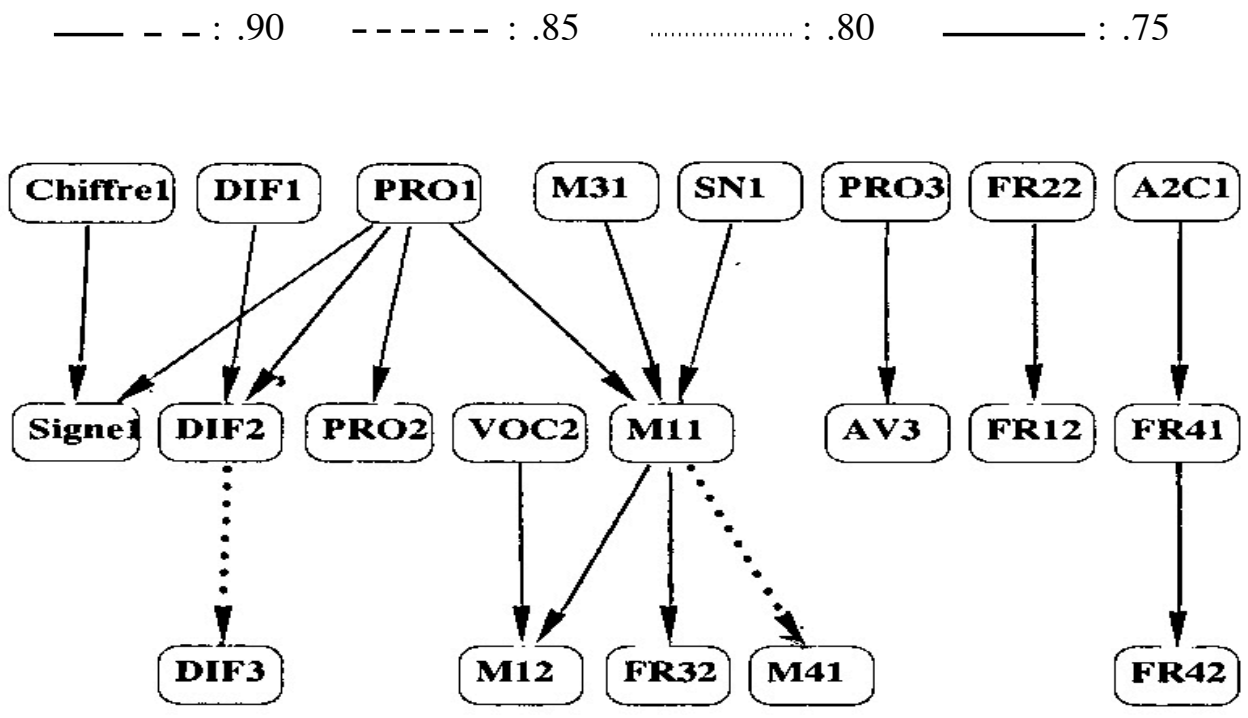

GRAPHE 7. Structure globale des implications entre les performances mesurées à trois occasions pour les élèves les plus performants à l'épreuve «!'Vocabulaire!»

Légende. Indices appliqués aux flèches!: 


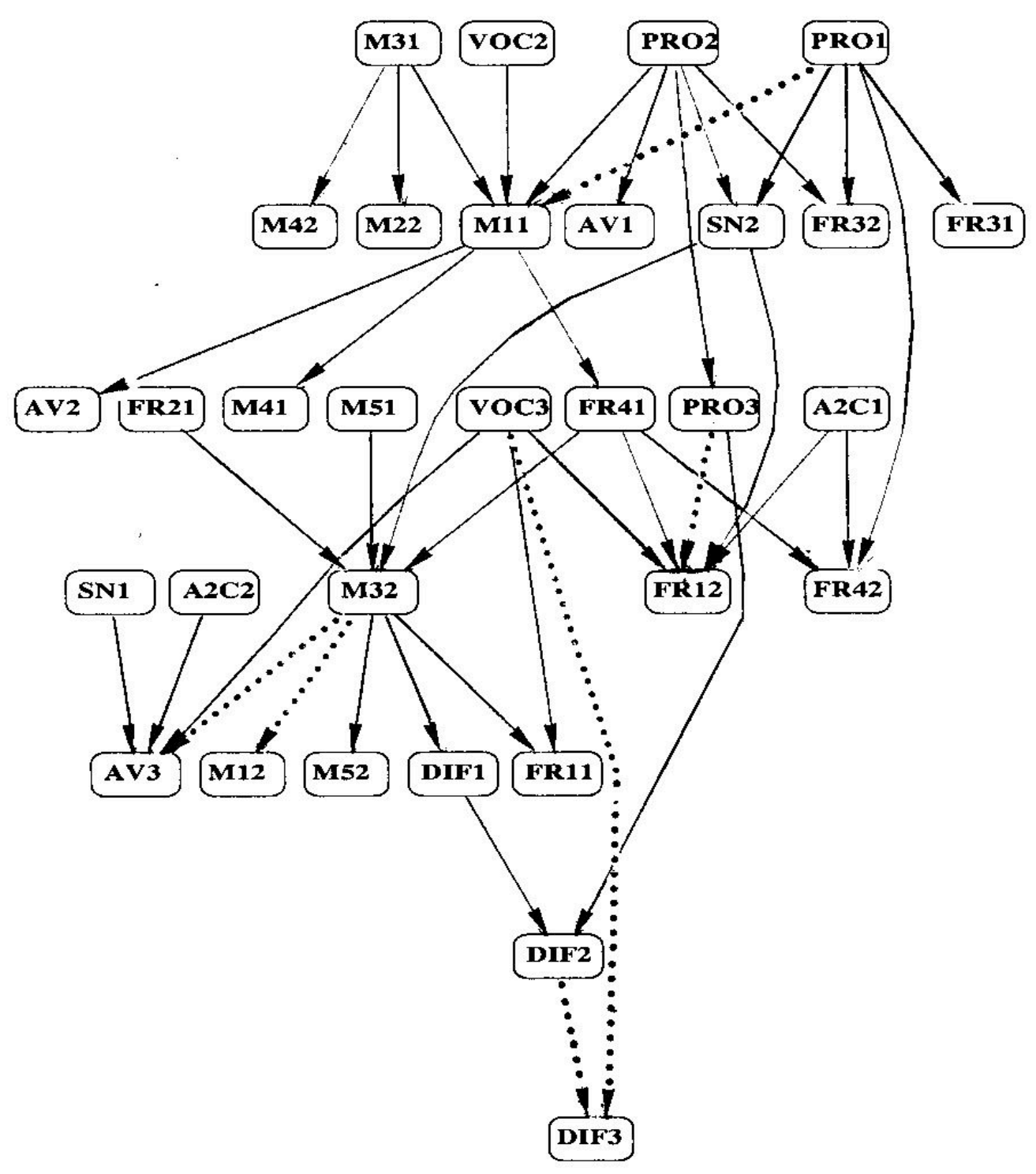

GRAPHE 8. Structure globale des implications entre les performances mesurées à trois occasions pour les élèves les moins performants à l'épreuve «!Vocabulaire!»

Légende. Indices appliqués aux flèches!:

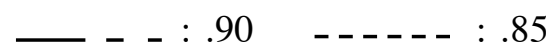

Que nous fournissent comme informations ces différentes structures, obtenues en appliquant, comme précédemment, les mêmes seuils, pour chacune d'elles, que ceux appliqués à la population totale ?

a) Des liaisons implicatives apparaissent pour le groupe complet mais n'apparaissent pas dans un ou plusieurs sous groupes. Exemple!: FR3 1 implique AV 3 apparaît au niveau du groupe complet mais dans aucun des sous-groupes.

b) Des liaisons implicatives apparaissent au niveau de sous groupes mais pas à celui du groupe complet. Exemple!: «Chiffres» 2 et A2C 1 implique «Images» 2 dans le groupe MAT-. 
c) Des implications différentes apparaissent entre sous groupes aux performances différentes sur la même variable. Exemple!: la relation implicative SN 2-> ${ }^{34}$ DIF 2 apparaît pour MAT+ mais pas pour MAT-!; La relation implicative PRO 2->SN 3 apparait pour MAT- mais pas pour MAT+. La relation implicative PRO 3->AV 3 apparaît pour VOC+ mais pas pour VOC-. La relation implicative PRO 2->AV 1 apparaît pour VOC- mais pas pour VOC+.

Pour illustrer de nouveau les considérations ci-dessus, examinons concrètement ce que cela peut signifier en ce qui concerne l'avant dernier exemple proposé, à savoir!: «PRO 2->SN 3 apparaît pour MAT- mais pas pour MAT+!».

Rappelons que SN correspond à l'épreuve!« Séries Numériques». Pour cette épreuve, à partir d'une série de chiffres et de nombres donnée $(« ! 2 / 12 / 4 / 9 / 6 / 6 / 8 / ! »)$, les exercices proposés visent à rechercher, parmi des couples de chiffres et de nombres proposés (8/10, 10/12, 10/11, 9/10, 9/11,3!/10), le couple qui continue la série (à savoir, ici, 3/10). L'épreuve PRO ("Proverbes ») consiste à trouver parmi 6 phrases («!1. Les souris ne sont pas méfiantes!», «!2. Quand le patron est parti, les apprentis s'amusent!», ... «!6. Les souris aiment danser!»), celle dont le sens se rapproche le plus du proverbe présenté au début de l'exercice considéré («!Quand le chat n'est pas là, les souris dansent!»). L'épreuve MAT ( "Matrices »), qui dissocie les deux groupes considérés selon le niveau de performance réalisé (Ceux qui réalisent des performances au delà de la moyenne du groupe et ceux qui réalisent des performances en deçà de la moyenne du groupe), est constituée de séries d'exercices présentés sous forme d'un carré à quatre cases. Les deux premières cases contiennent chacune un dessin (par exemple!: un rond blanc et un rond noir ou un losange avec un point et un losange sans point). La première des deux cases suivantes, placées sous les deux premières, contient un troisième dessin (par exemple!: un carré blanc ou un carré avec un point). Le but de l'exercice consiste à trouver, pour la quatrième case, un dessin qui présente les mêmes rapports au troisième dessin que le $2^{\mathrm{e}}$ dessin fait valoir avec le premier (Il résulte de cette règle que les réponses aux exemples simples proposés sont un carré noir ou un carré sans point). Pour les élèves qui ont de faibles performances à cette dernière épreuve (MAT-), un niveau de maitrise de la compréhension verbale impliquerait un niveau de performances à l'épreuve «Séries Numériques » (SN) supérieur ou au moins égal, ce qui ne serait pas le cas pour les élèves ayant de bonnes performances à l'épreuve «!Matrices!» (MAT+).

L'explication interprétative qui peut être avancée ici consiste à évoquer un possible phénomène compensatoire à la faiblesse de la maîtrise de l'analogie sur données figurées (MAT) (ou des fonctionnements cognitifs qui sous-tendent cette maîtrise) se réalisant par une bonne maîtrise du raisonnement sur données numériques (SN) (ou des fonctionnements cognitifs qui sous-tendent cette maîtrise) dans le développement de la maîtrise de la compréhension verbale (PRO).

d) Des relations implicatives différentes apparaissent dans des groupes aux performances comparables $^{35}$ mais portant sur des variables différentes. La relation implicative A2C 1->SN 3->AV 3 apparaît dans MAT + mais pas dans VOC + . La relation implicative PRO 1->SN 2->M3 2->DIF 1 apparaît dans VOC - mais pas dans MAT-. La relation implicative «Chiffres» 2-> «Images» 2 et A2C 1->«Images» 2 apparait dans MATmais pas dans VOC-.

e) Des liaisons communes apparaissent dans tous les groupes, comme, par exemple, les relations implicatives DIF 1->DIF 2->DIF 3, mais à différents niveaux de chaque sous structure.

\footnotetext{
${ }^{34}$ SN 2 implique DIF 2.

${ }^{35}$ Les deux groupes considérés ayant des performances soit inférieures, soit supérieures, à la moyenne des performances du groupe de référence.
} 
f) Certaines variables n'apparaissent dans aucun sous-groupe, comme, par exemple, «Mots» 1 , «Signes» 2.

Pourquoi certaines liaisons implicatives apparaissent ou n'apparaissent pas pour la population globale, au regard des sous groupes et vice versa!? Pourquoi ces liaisons apparaissent pour des sous populations de la population globale et n'apparaissent pas pour d'autres sous populations, et vice versa!? Une première raison explicative peut être donnée. Les mêmes liaisons implicatives existent mais pour des seuils retenus différents de ceux choisis de manière standard pour tous les groupes. La question se pose alors de la valeur qui peut être accordée à des seuils retenus très bas. Et il peut exister des cas de figure pour lesquels la liaison n'existe effectivement pas.

\section{QUESTIONS MÉTHODOLOGIQUES POSÉES, ESSAIS D'INTERPRÉTATIONS PARTIELLES}

\subsection{QUESTIONS MÉTHODOLOGIQUES LIÉES À L’ANALYSE FORMELLE DU MODÈLE CONSIDÉRÉ}

De manière plus large que signifie réellement chaque résultat obtenu et présenté au point 3 ?

\subsubsection{Le principe même de l'analyse implicative}

Pour mieux en saisir le sens il convient de revenir sur le principe qui fonde l'analyse implicative $^{36}$. À partir d'un prédicat!défini, par exemple : «la lampe T ne peut s'allumer que si la lampe U est allumée ${ }^{37}$, l'implication peut être formulée de la façon suivante!: « si la lampe T est allumée alors la lampe $U$ est allumée » (ou «la lampe T allumée $(P)$ implique la lampe $U$ allumée $(Q) » !$ : modus tollens, $\mathrm{P}|| \mathrm{Q})$.

Mais la relation implicative peut être vraie du point de vue de la logique et dépourvue de sens voire absurde dans la réalité concrète de l'observateur ${ }^{38}$. Une relation implicative a du sens dans la réalité observée, s'il existe un contexte ${ }^{39}$ qui le valide, une homogénéité minimale de contenu, ou, a minima, une logique d'appartenance à un même domaine (une même classe d'observables), dans lequel une relation triviale ou théorique de dépendance entre $\mathrm{U}$ et $\mathrm{T}$ est reconnue et validée selon d'autres critères de la réalité vécue par l'observateur. Par exemple, dans le domaine scolaire, dont il est question dans cet article, savoir faire des problèmes d'arithmétique $(\mathrm{P})$ nécessite sans aucun doute, de maîtriser a minima!: «!savoir faire des opérations!» (q1) et «!connaître le sens des opérations!» (q2). Il est vraisemblable, dans ce cas, que des scores élevés à des évaluations effectuées dans ces deux domaines (q1 et q2) seront sensés exister avant qu'apparaisse un score élevé à une évaluation portant sur des problèmes d'arithmétique $(\mathrm{P})$. P || Q (q1+q2) signifie que, et ici sous l'angle des scores correspondant aux performances réalisées (fortes!: $+{ }^{40}$ ) ou quasiment non réalisées (faibles!: - $), \mathrm{Q}+(\mathrm{q} 1+$ et $\mathrm{q} 2+)$ et $\mathrm{P}$ - correspond bien à une

\footnotetext{
${ }^{36}$ Cf. la définition développée p. 54.

${ }^{37}$ C'est le cas de figure lorsque des lampes sont mises en série sur un circuit électrique.

${ }^{38}$ C'est le cas, par exemple, d'une formulation telle que la suivante!: " si les peupliers sont des arbres alors l'Afrique est un continent ».

${ }^{39}$ Renvoyant à des rapports de sens entre propositions!: une observation courante tirée de l'expérience concrète, les lois de la physique appliquées à un circuit électrique simple, etc.

${ }^{40}$ Soit en équivalent logique $P(\mathrm{P}+)$ opposé à non $P(\mathrm{P}-)$.
} 
situation susceptible d'être observée, tout comme Q-(q1- et q2-) et $\mathrm{P}-$, ainsi que Q+(q1+ et $\mathrm{q} 2+)$ et $\mathrm{P}+$. Par contre rencontrer $\mathrm{Q}-(\mathrm{q} 1-$ ou/et $\mathrm{q} 2-)$ et $\mathrm{P}+$ ne paraît guère plausible.

Cependant, la logique implicative formelle, au sens du logiciel CHIC, si elle peut se satisfaire du comparatif ordonnancé correctement, entre scores quantitatifs reflétant des performances relevant de cadres de pensée incertains, voire problématiques, ne permet pas d'analyser, totalement, et avec clarté, sous cet angle de la performance, et ceci de manière qualitative, les logiques d'implication strictes, au sens de la causalité, au regard du contexte et des contenus traités. Sens de la causalité qui lui donnerait, dans le «lréel!» abordé, toute sa signification.

En ce qui concerne les acquis scolaires, les performances peuvent renvoyer à des modèles intégratifs des savoirs, qui sur un plan conceptuel, sont relativement étayés et constituent des «!outils explicatifs acceptables!».

Mais, traiter des acquisitions scolaires, sur la base des épreuves d'évaluation réalisées en classe de $6^{\mathrm{e}}$, avec l'outil «!analyse implicative!» basé sur la comparaison de performances sur les différents domaines considérés, ne constitue pas la méthode la mieux adaptée à la situation abordée. Elle permet, tout au moins, de confirmer, ou d'infirmer, des inférences hypothétiques au départ.

Il n'en est pas de même lorsqu'il s'agit d'éléments théoriques encore mal ou faiblement conceptualisés, comme c'est le cas pour les capacités cognitives, dans le cadre d'analyses confirmatoires (ou non), ou, de manière encore plus discutable, de recherches, complètement exploratoires, de structures à partir desquelles sont inférés des modèles explicatifs des conduites et comportements. Ceci est pratiquement le cas des travaux dont il est question dans cet article, relevant, en particulier, d'une psychologie du développement dont les conceptions théoriques susceptibles d'en rendre compte sont encore très largement discutées. Savoir lire l'heure nécessite-t-il de maîtriser un certain nombre d'exercices psychomoteurs d'équilibre ${ }^{41}$ ? Voilà, parfois, la situation du chercheur dans un domaine, le développement cognitif, ou malgré les affirmations plus ou moins péremptoires affichées, les résultats de la recherche, les modèles théoriques proposés ${ }^{42}$, ne sont pas nécessairement à la hauteur des espoirs que le chercheur peut y mettre.

Pourtant le rapport entre la mesure portant sur une série d'exercices en rapport avec l'équilibre $(\mathrm{Q})$ et la mesure de la capacité à lire l'heure $(\mathrm{P})$ peut très bien fournir des résultats satisfaisants au plan de l'analyse implicative $(\mathrm{Q}+/ \mathrm{P}+, \mathrm{Q}-/ \mathrm{P}-, \mathrm{Q}+/ \mathrm{P}-$ souvent observé, $\mathrm{Q}-/ \mathrm{P}+$ pas, ou rarement observé). Le psychologue, au regard de la forte hétérogénéité entre les domaines considérés, se contentera de dire prudemment que la performance en $\mathrm{Q}$ "précède » la performance en $\mathrm{P}$. Mais n'en infèrera pas pour autant que la capacité à lire l'heure (P) implique, pour l'enfant, une certaine maîtrise de l'équilibre $(\mathrm{Q})^{43} \ldots$

Pour tous chercheurs, il est depuis longtemps, clair que la valeur de l'outil tient essentiellement à la qualité du contexte théorique, méthodologique dans lequel il est appliqué. La pratique consistant à utiliser les modèles statistiques structuraux dans le cadre d'analyses exploratoires est donc toujours hasardeuse, voire suspecte, notamment à cause d'artefacts grossiers possibles. Une recherche utilisant ces modèles dans le cadre

\footnotetext{
${ }^{41}$ Marcher sur une poutre, rester en équilibre, sur un plot en hauteur, sur un pied, etc.

${ }^{42}$ Ici, la théorie piagétienne, les théories néo piagétiennes, les conceptions cognitives du traitement de l'information, etc.

${ }^{43}$ Soit $\mathrm{P} \sqcup \mathrm{Q}$
} 
d'analyses confirmatoires, à la suite de travaux comparables déjà réalisés, cohérente avec d'autres recherches portant sur d'autres aspects du même domaine, et s'inscrivant dans un cadre théorique bien cerné dans la littérature, constitue bien davantage un ensemble contextuel méthodologiquement plus acceptable. Il en serait de même de résultats obtenus par la recherche, sur la base de conceptions précisément spécifiées et confrontées à un jeu d'hypothèses fortes.

Les travaux présentés ici renvoient-ils à la notion de «!précédence ${ }^{44}$ !» évoquée cidessus ou permettent-ils de valider effectivement la relation implicative!(la performance Q est nécessaire à la performance $\mathrm{P}$ )!? Le traitement de cette question peut nécessiter de longs développements. Ne seront traités, ici, seulement, que quelques points méthodologiques parmi les plus évidents à aborder.

Au-delà de cette question de précédence, la relation implicative, dans son acception la plus forte, renvoie à la recherche de relations causales entre différents évènements, au sens du syllogisme classique, tel que cela peut apparaître, par exemple, dans la formulation suivante!: "les toits sont mouillés alors il a plu » ${ }^{45}$. Mais la relation implicative, au sens de la causalité, telle que développé dans le cadre de cet exemple, certes anecdotique, dépend, ici encore, du contexte, elle est donc nécessairement "située ». Il en va souvent très différemment dans le domaine des sciences humaines. Le cadre théorique restant largement hypothétique, on peut tout au plus dire, dans un contexte, au moins, pour partie, indéterminé, qu'il y a "précédence » d'un évènement par rapport à un autre et non pas nécessairement rapport de causalité. Encore faut-il savoir de quelle précédence il est question!?

\subsubsection{La question de la «précédence »}

a) Il existe différentes conceptions de la «!précédence!»!; quels sont leurs rapports avec l'implication tel que défini dans le logiciel CHIC

Il existe un premier état formel de précédence dans le temps lorsqu'une performance qui n'apparaissait pas, à un moment donné, pour une variable, apparaît quelque temps plus tard. Par exemple, et ceci de manière évidente, pour un sujet observé, l'absence de la capacité à lire l'heure précède l'expression ultérieure de cette capacité (non X précède $\mathrm{X}$ ).

Mais il est aussi d'observation courante, de repérer la succession dans le temps entre différents évènements. Cette observation traduit une autre forme de précédence. L'évènement $(\mathrm{X})$ apparaît à un moment donné, puis l'évènement $(\mathrm{Y})$ ultérieurement, l'évènement $(\mathrm{X})$ restant présent (ou pas). La précédence est, ici, constituée par une succession d'évènements différents (X précède $\mathrm{Y}$ ). L'inversion de la succession des évènements introduisant l'émergence de phénomènes cycliques ${ }^{46}$ (X précède $\mathrm{Y}$ qui précède $\mathrm{X})$.

La précédence peut aussi relever d'une observation de combinaison de situations observées à un moment donné dans le temps pour un groupe de sujets. C'est le cas de figure traduit par la relation "implicative » $(\mathrm{A}->\mathrm{B})$ développée dans le logiciel $\mathrm{CHIC}$. Dans ce cas, l'évènement $(B)$ précède l'évènement $(A)$ lorsque $(A)$ et $(B)$ sont présents ou absents ensemble, ou lorsque (B) est présent en l'absence de (A), alors que l'évènement

\footnotetext{
${ }^{44}$ Une performance en précède une autre dans le temps.

${ }^{45} \mathrm{Ou}$ bien, si la lampe $\mathrm{T}$ s'allume alors il faut que la lampe U soit allumée (comme évoqué précédemment à propos de la mise en série, sur un circuit électrique, de lampes).

${ }^{46}$ Semblable à l'alternance d'évènements, comme l'obscurité puis la lumière du phare qui éclaire l'océan.
} 
constitué par la présence de (A) sans la présence de (B) n'apparaît pas (B et non A, A et B, non $\mathrm{A}$ et non $\mathrm{B}$, vrai!; non $\mathrm{B}$ et $\mathrm{A}$, faux).

Nous pouvons, ainsi, concevoir qu'une performance traduisant l'expression d'une capacité en précède une autre sous l'angle de ce qui est évoqué ci-dessus, mais cela n'induit pas ou pas nécessairement qu'une capacité maîtrisée et disponible pour un sujet est nécessaire ${ }^{47}$ à une autre, et encore moins qu'elle peut être la cause même de l'expression de cette autre capacité.

b) Précédence ne veut donc pas dire nécessairement implication!: cela dépend d'un modèle fort de traitement des interactions entre variables plus ou moins hétérogènes et/ou du caractère homogène des données ${ }^{48}$.

Comme cela a été indiqué précédemment ${ }^{49}$, ce n'est pas seulement la mesure et son traitement qui caractérise l'implication, mais bien davantage le modèle ou l'aspect théorique traité. Dans le cadre de l'exemple développé précédemment, au plan du développement de l'enfant, la maîtrise de certaines formes d'équilibre psychomoteur «!précèdent dans le temps!» la capacité à «!savoir lire l'heure!». L'état des performances rend simplement compte, dans le cadre de l'analyse implicative, de la plus grande difficulté à mettre en évidence une performance à un âge donné dans un domaine précisé (savoir lire l'heure) alors que la performance peut être établie sur l'autre (différents exercices d'équilibre). La performance, au regard de la variable considérée initialement, "précède ", là encore, une quelconque performance portant sur l'autre variable, sans pour autant que soit établie une relation d'implication, au sens d'une nécessité préalable ou d'un rapport de cause à effet. Cependant, en termes de développement psychologique du sujet, le résultat vaut, mais sous des aspects très simples. L'enfant maîtrise une habileté avant de maîtriser l'autre sans que l'on puisse, dans le cas de figure évoqué, établir une relation de causalité de l'une par rapport à l'autre.

Nous pouvons aussi dire la même chose d'une autre manière. A un âge donné, l'épreuve portant sur la maîtrise de la lecture de l'heure est plus difficile, pour les enfants considérés, que l'épreuve choisie, portant, elle, sur la maîtrise de l'équilibre. Ceci se traduit bien pour un groupe d'enfants de différents âges, par les résultats théoriques suivants. Les enfants les plus jeunes réussissent des exercices de l'épreuve «!équilibre!» et aucun de l'épreuve «!lire l'heure!». À un âge intermédiaire, les enfants réussiront la plupart des exercices d'équilibre qui leur seront présentés (par exemple, 80 à $100 \%$ ) et quelques exercices portant sur la lecture de l'heure (par exemple, 0 à $20 \%$ ). À un âge plus avancé, la plupart des enfants réussiront tous les exercices des deux épreuves proposées. Les deux points de vue portant sur la "précédence $»^{50}$ peuvent fonctionner conjointement, donc rendre compte, de manière très complète, de la logique «limplicative!», sans que cette précédence renvoie à une causalité articulant la performance définie à partir d'une épreuve à la performance définie à partir d'une autre épreuve. En ce sens et en ce qui concerne les épreuves de la recherche engagée, la conclusion peut être la même!: des épreuves à un âge donné sont plus difficiles que d'autres qui les "précèdent $»^{51}$. C'est la question des

\footnotetext{
${ }^{47}$ C'est-à-dire : fait partie de ce qui peut en constituer la cause parce que «!nécessaire à!», mais là aussi pas nécessairement la cause au sens fonctionnel du terme, comme maîtriser le sens des opérations fait partie de ce qui est nécessaire à une efficacité à maîtriser des problèmes d'arithmétique mais pas forcément (ou uniquement) la cause...

${ }^{48}$ Observables, par exemple, sur les mêmes contenus, ou, a minima, de la catégorie (semblable ou relatif à un ensemble fonctionnel) de contenus, traités successivement dans le temps.

${ }^{49} \mathrm{Cf}$. ci-dessus début du point 4.1.1.

${ }^{50}$ Performance successives dans le temps ou logique «!implicative!» à l'instant $t$.

${ }^{51}$ Ce qui inclut le deuxième point de vue de la précédence évoquée!: «!à un âge intermédiaire, la plupart
} 
rapports établis formellement au plan théorique de l'épreuve plus difficile à l'épreuve la plus facile qui permet de poser un résultat autre que trivial!: une épreuve est plus difficile que l'autre. Pour mieux comprendre il convient d'évoquer un nouvel exemple relevant de l'évidence. Supposons que l'on veuille mettre en avant que la maîtrise du vocabulaire «!précède!» la maîtrise de la compréhension de textes lus. Une épreuve de vocabulaire portant sur la définition de mots choisis dans ceux qui sont sensés être acquis dans les premières années de l'enseignement élémentaire est construite à cet effet. Il s'agit, par exemple, pour chaque mot présenté de choisir sa définition parmi plusieurs définitions proposées. Une épreuve portant sur la maîtrise de textes lus $\mathrm{s}^{52}$ est constituée à partir d'extraits d'ouvrages de la littérature étudiés en classe de seconde des lycées. Il est vraisemblable que les résultats obtenus iront dans le sens des hypothèses formulées concernant la précédence de la maîtrise du vocabulaire par rapport à la maîtrise de la compréhension des textes lus sans qu'il y ai de rapport implicatif direct entre les mots de vocabulaire et les textes lus. Une même conclusion peut exister à l'inverse ou entre deux épreuves de vocabulaire ou de compréhension de textes lus. En fait la seule conclusion valide qui peut être, en toute rigueur, tirée des observations est qu'une des épreuves ${ }^{53}$ est plus difficile que l'autre. Etablir cependant par l'analyse implicative entre deux épreuves qu'une épreuve est d'un niveau de difficulté supérieur à l'autre n'est pas nécessairement négligeable comme résultat, quand le différentiel de niveau de difficulté des deux épreuves n'est pas aussi évident que pour l'exemple traité ci-dessus.

Mais, alors, comment traiter, dans le sens souhaité, des résultats obtenus à propos des épreuves proposées dans la recherche présentée ici!? La relation «d'implication» entre l'épreuve «proverbes » et l'épreuve "vocabulaire », ou entre un des domaines retenus de l'évaluation «français» ou «mathématiques » et un sub-test de l'échelle collective de niveau intellectuel (ECNI), tient-elle à une condition nécessaire du type «il faut maîtriser $B$ pour pouvoir maîtriser $A »^{54}$, ou simplement au fait que l'épreuve de «type » $B$ présente des exercices plus faciles à traiter que l'épreuve de «type » $A$ !?

Tout ceci est bien loin d'une utilisation de l'analyse implicative susceptible d'articuler causes et effets entre plusieurs variables, rendant compte, dans le domaine de la psychologie de la structuration «!constructiviste $!^{55} »$, en particulier des variables relevant de la sphère cognitive.

En première analyse, seules des conclusions de plus (ou moins) grande difficulté des épreuves pour une tranche d'âge donnée et une population d'élèves donnée, peuvent être retenues. Dans le même ordre d'idée, la relation implicative, «!paradoxale!» du point de vue des résultats attendus (cf. § 3.2, notamment p. 58), entre mesures successives de performances aux trois occasions, pour la même variable, est bien observée (plus ou moins directement). Par exemple, et le plus souvent, contrairement à une forme possible de relation intuitivement attendue, "une performance initiale à un certain niveau est nécessaire pour permettre la performance suivante », la relation observée est du type!: «PRO 1 implique PRO 2 qui implique (plus ou moins indirectement) PRO 3 ». Cela veut simplement dire que l'on observe, le plus souvent des performances supérieures à la seconde et à la troisième occasion et plus rarement l'inverse. Pour autant une performance minimale, initiale, en $t 1$, peut être nécessaire à la progression des performances en $t 2$ et $t 3$ !

\footnotetext{
des performances à l'une des épreuves sont inférieures aux performances obtenues à l'autre épreuve!».

${ }^{52}$ Par exemple répondre à des questions précises portant sur un texte proposé.

${ }^{53}$ Certes hétérogènes entre elles par leurs contenus et leurs modalités de mise en œuvre.

${ }^{54} \mathrm{Ou}$ «!la maîtrise de $\mathrm{B}$ est nécessaire à la maîtrise de $\mathrm{A}$ !».

${ }^{55}$ En référence, par exemple, dans le domaine du développement psychologique de l'enfant, aux travaux de J. Piaget.
} 
L'observation faite ci-dessus résulte, ici, simplement du fait que les enfants, globalement, progressent d'une passation à l'autre dans la maîtrise du domaine dans lequel sont effectuées les mesures successives. Ceci ne constitue pas pour la recherche considérée une information très pertinente. Dans une situation pédagogique, les résultats peuvent attester de la progression des apprentissages, mais pour cela l'analyse implicative n'est peut-être pas nécessaire à l'enseignant, au pédagogue, pour qu'il se rende compte des évolutions, dans le domaine considéré, des apprentissages effectués par ses élèves...

Des épreuves de différents niveaux de difficulté, une progression significative d'une passation à l'autre pour une épreuve donnée, mais aussi une progression plus importante pour une épreuve par rapport à une autre, d'une occasion à une autre, telles peuvent, donc, être les explications susceptibles de rendre compte de l'ordonnancement des données, au sens de l'analyse implicative, et portant sur l'ensemble des mesures (cf. Graphes 4 et 9), sans pour autant pouvoir faire valoir une quelconque implication d'un observable par rapport à l'autre. Par contre, un schéma implicatif cohérent, et ici pertinent du point de vue des intentions du chercheur, pourrait être!: «!une performance sur la variable A implique une performance sur la variable $\mathrm{B}$ si une performance élevée ${ }^{56}$ à $\mathrm{B}(\mathrm{B}+)$ en $t 1$ est associée à un accroissement de performances mesuré sur la variable $\mathrm{A}$ de $t 1$ à $t 2$ et non pas l'inverse ${ }^{57}$.

c) Les rapports entre précédence et implication peuvent être plus ou moins complexes notamment en ce qui concerne la précédence au sens strict, c'est-à-dire temporelle, et l'implication au sens du logiciel CHIC

Il va être traité, plus particulièrement dans ce point et de manière plus approfondie, du sens qu'il convient de donner aux relations implicatives, dégagées par l'analyse statistique du logiciel CHIC, entre une performance réalisée à la première occasion $(\mathrm{t} 1)$ ou à la seconde occasion (t2) par rapport à une performance, pour une autre variable, réalisée à la deuxième (t2) ou à la troisième occasion (t3)58. Dans le langage relatif au modèle logicomathématique du traitement statistique proposé, la performance en note standard59 obtenue à l'épreuve vocabulaire à la première occasion «!implique!» la performance obtenue selon les mêmes règles à l'épreuve appartenance à deux classes à la troisième occasion. Autrement dit!: des résultats plus élevés, en notes standards, à l'épreuve appartenance à deux classes peuvent être associés à des résultats plus faibles à l'épreuve vocabulaire, mais l'inverse n'apparaît pas ou suffisamment rarement pour être considéré comme négligeable.

Ceci peut nous amener à renforcer, en première approximation, un point de vue, faire une inférence, qui tendrait à considérer qu'il est nécessaire d'avoir des performances élevées dans la maîtrise des opérateurs logiques (épreuve appartenance à deux classes) afin de pouvoir maîtriser le sens des mots (épreuve vocabulaire).

Pourtant d'autres conclusions sont envisageables. Par exemple, l'épreuve vocabulaire est, au regard des scores réalisés par les élèves, particulièrement difficile à l'âge considéré ce qui amène à observer que des performances plus élevées à cette épreuve

\footnotetext{
${ }^{56}$ Supérieure à la moyenne des performances du groupe de référence.

57 Soit $\mathrm{B}+$ /acc. A+, B-/acc. A-, B+/ acc. A-, vrais!; mais B-/acc. A+, faux (relation implicative «!cause/effet!»). Ou bien s'il s'agit d'une formulation associative, dans un sens qui peut apparaître encore plus stricte (corrélation)!: B+/acc. A+, B-/acc. A-, vrai!; mais B+/ acc. A-, B-/acc. A+, faux.

${ }_{58}$ Cf. par exemple, dans le Graphe 3!: VOC 1 implique A2C 3, A2C 1 implique SN 3, M1 2 implique AV 3.

${ }^{59}$ Ici pourcentage d'exercices réussis par rapport au total des exercices proposés.
} 
ne sont pas, ou peu, associées à des performances faibles à l'épreuve appartenance à deux classes alors que l'inverse est plus souvent le $\operatorname{cas}^{60}$.

Autre cas de figure possible, à la différence de l'épreuve vocabulaire, la progression concernant l'épreuve appartenance à deux classes entre les mesures effectuées, est très importante ce qui amène l'ordonnancement des niveaux de performance, en note standard, traduisant une implication de fait, d'une occasion à une autre, entre les variables considérées et au seuil choisi pour le traitement des données par le logiciel.

En l'absence d'éléments théoriques méthodiquement structurés, et du fait du choix méthodologique de performances, quantitativement mesurées, en évolution, il paraît difficile de choisir entre les différentes explications, en particulier de confirmer la relation implicative, au sens de la causalité, évoquée ci-dessus. Tout au plus, les données, à ce niveau de leur traitement, ne valident pas l'hypothèse inverse qui consisterait à considérer qu'il est nécessaire de maîtriser le vocabulaire pour maîtriser, sur contenu verbal, des opérateurs logiques (appartenance à deux classes).

\subsection{TRAITEMENT DES QUESTIONS MÉTHODOLOGIQUES AU REGARD DES RÉSULTATS OBTENUS}

\subsubsection{L'implication au sens logique peut ne pas renvoyer à la causalité}

À propos du dernier aspect soulevé ci-dessus, une analyse plus complète des données amène, d'ailleurs, à revoir, plus largement, ce point de vue ambitieux d'une possible relation de causalité. En effet, pour que cette hypothèse implicative puisse avoir ce sens il est nécessaire qu'elle soit cohérente avec d'autres. Si PRO 2 implique VOC $3^{61}$ (cf. Graphe 9 ci-dessous), alors PRO 2 implique VOC 2, et ce qui renforcerait la cohérence implicative $^{62}$ PRO 1 implique VOC 1, PRO 3 implique VOC 3, et peut-être même que PRO 1 implique VOC 2, PRO 1 implique VOC 3. Or une simple observation du Graphe $9^{63}$ permet de faire valoir que VOC 2 implique PRO 2 qui implique d'ailleurs bien VOC 3 , et VOC 3, même indirectement, implique PRO 3, à savoir la position inverse, c'est-à-dire qu'une bonne maîtrise du vocabulaire passe par une bonne maîtrise, au départ, de la compréhension de textes lus. Par ailleurs, aucune relation implicative aux seuils choisis n'apparaît entre VOC 1 et PRO 1.

\footnotetext{
${ }^{60}$ À côté de résultats communs obtenus aux deux épreuves également faibles ou également forts.

${ }^{61}$ Une bonne maîtrise de la compréhension de textes lus implique la maîtrise du vocabulaire.

${ }^{62}$ Mais valide aussi la permanence de la difficulté différentielle des deux épreuves...

${ }^{63}$ Reprise dans les analyses systématiques à chaque occasion temporelle de prise de mesure pour l'ensemble des sub-tests de l'échelle collective de niveau intellectuel (ECNI) (cf. Graphes 1, 2, 3).
} 


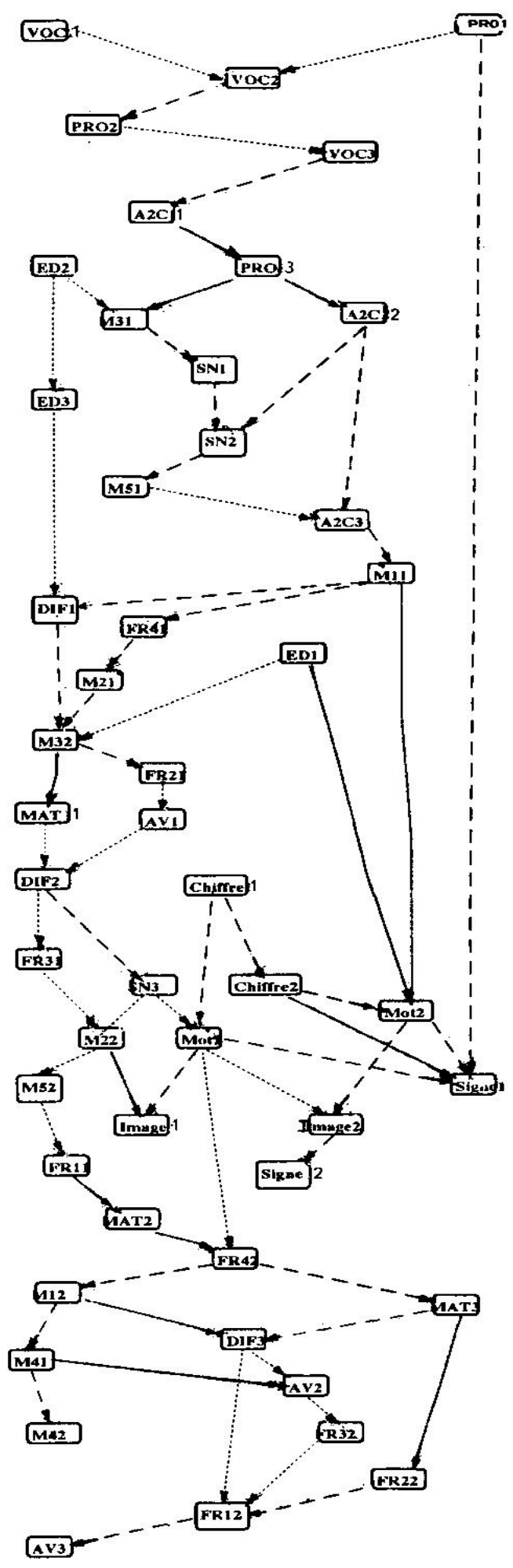

GRAPHE 9. Structure globale des implications entre les performances mesurées à trois occasions!: observations faites aux seuils $.90, .70, .65, .64$.

Légende. Indices appliqués aux flèches!: 
L'explication, là aussi, peut être simple. Elle renvoie, sur deux variables, à l'effet, déjà évoqué, de la progression des performances, plus ou moins fortes, pour l'ensemble des mesures considérées. En effet, une performance à la seconde occasion, entre variables, est habituellement supérieure, et ici le plus souvent, à celle correspondant à la première occasion. Ceci vaut, toutefois, entre deux variables différentes considérées, seulement si les difficultés des épreuves sont comparables (également faciles ou difficiles), pour les sujets considérés. La relation implicative reconnue comme abusive entre PRO 2 et VOC 3 tient au même phénomène que l'implication observée dans les faits entre, par exemple, PRO 1 et PRO2. PRO 1 implique PRO 2 dans la mesure où nous trouvons peu de performances PRO 1 associées à des performances inférieures à PRO 2, alors que l'inverse ${ }^{64}$ est vrai du fait d'une progression générale des performances, pour chaque élève considéré.

Une relation d'implication observée entre variables tient donc ici à une application inadéquate de l'analyse à des situations ordonnancées dans le temps qui amène à des conclusions erronées, en termes de relations causales éventuelles, du fait de l'artefact introduit par la progression temporelle des performances des sujets considérés. Cela veutil dire que seule la comparaison vaudrait pour des performances observées à un temps $t$ donné ? Pas nécessairement, nous pouvons avoir des performances faibles à une épreuve systématiquement associée à des performances plus élevées à une autre épreuve. Mais l'ordonnancement des données conforme au schéma de la relation implicative ne se résume pas nécessairement à la question de la difficulté d'une épreuve par rapport à une autre.

En effet, dans le cadre de l'implication stricte, A implique $\mathrm{B}$, et pour différents sujets, des performances élevées à la première épreuve peuvent être associées à des performances élevées à la seconde $(\mathrm{A}+$ et $\mathrm{B}+)$, à côté, pour d'autres sujets, de performances faibles aux deux (A- et $\mathrm{B}$-), ainsi que des performances faibles à la première sont associées, pour un troisième groupe de sujets, à des performances élevées à la seconde $(\mathrm{A}-$ et $\mathrm{B}+)$. Seules des performances élevées à la première associées à des performances faibles à la seconde (A+ et B-) n'apparaissent pas, ou quasiment pas, dans les observations effectuées. Il s'agit là d'une situation, tout de même bien particulière, au regard d'une situation plus courante de différence de difficultés entre épreuves (A plus "difficile » que B), attestée par les notes standards, s'inscrivant dans une logique présentant un jeu d'occurrences différentes plus réduites (A- et $\mathrm{B}-\mathrm{ou} \mathrm{A}-\mathrm{et} \mathrm{B}+$ fréquent, $\mathrm{A}+$ et $\mathrm{B}+$ comme $\mathrm{A}+$ et $\mathrm{B}$ - rare).

Dans le cas de figure traité, l'hypothèse implicative qui paraît la plus conforme ${ }^{65}$, aux faits observés, sans pour autant être totalement assurée, est que la maîtrise du vocabulaire implique plutôt, à ce niveau du développement de l'enfant (classes de $6^{\mathrm{e}}$ et $5^{\mathrm{e}}$ des collèges), une bonne maîtrise de la compréhension en lecture. Pour le moins, cette observation mériterait d'être étayée par des résultats plus clairs que ceux obtenus ici. Il resterait à clarifier l'éventuelle confusion, susceptible d'exister, entre les deux formes de «!précédence!» ici traitées conjointement. La précédence temporelle vaut si la maîtrise d'une habileté en précède une autre dans le temps et au cours du développement de l'enfant. La précédence conditionnelle, possible, apparaît entre deux variables si une performance n'émerge des résultats que si une performance au moins égale, en note standard, pour une autre variable lui est associée et pas l'inverse. Les deux, chacune pour elle-même, peuvent aboutir dans un cadre théorique structuré et sur la base d'hypothèses

\footnotetext{
${ }^{64}$ C'est-à-dire!: la performance à PRO 2 est assez systématiquement associée à une performance inférieure à PRO 1.

${ }^{65}$ L'objection du niveau de difficulté différent entre les deux épreuves restant pertinente.
} 
fortes à valider une possible relation implicative, au sens d'une relation causale, entre deux variables, sous réserve!: de bien clarifier le contexte dans lequel ces variables sont mises en relation, de préciser les références théoriques convoquées, le champ des résultats déjà obtenus, et de fournir les résultats de différentes contre épreuves confirmant la robustesse de l'hypothèse avancée face aux faits observés.

4.2.2. Les structures implicatives partielles (observées sur des sous-groupes de la population totale) sont-elles incluses ou non dans la structure implicative globale?

La réponse sur ce point est négative puisque des relations implicatives, pour des sousgroupes (cf. par exemple!: les liaisons correspondant au sous-groupe MAT- évoqué au point b) p. 67), n'apparaîssent pas dans les relations observées, pour le groupe global d'appartenance.

\subsubsection{La question des différentes structures apparaissant aux différentes occasions}

Sur ce point, plusieurs hypothèses peuvent être formulées. Deux seront principalement retenues ici. Une première hypothèse est méthodologique. Même si les épreuves sont constituées pour traiter d'une certaine fidélité dans la mesure, celle-ci n'est pas à l'abri, surtout à une telle distance temporelle, de fluctuations possibles et fortes de la mesure. Le fait de prendre les scores bruts, même traduits en pourcentages du score maximum possible, et non pas les notes standards ${ }^{66}$, accentue la difficulté. Les structures implicatives différentes aux différentes occasions pourraient tenir, pour une partie des résultats au moins, simplement aux fluctuations différentielles non négligeables de la mesure. La seconde hypothèse possible, la plus intéressante pour le psychologue, mettrait en avant, sur ce plan du psychologique, une particularité possible du développement. Les relations implicatives entre les variables changeraient au fil de l'évolution dans le temps des sujets considérés.

\subsubsection{La question des différentes structures en fonction des sous populations considérées}

Cette question n'est pas sans poser de délicates questions sur les rapports multiples qui peuvent exister entre les différentes dimensions, en fonction des caractéristiques des sujets considérés. Il est tout à fait vraisemblable que les différences observées renvoient à une certaine vicariance d'expressions et d'évolutions de sous groupes d'élèves en fonction de spécificités qui leur sont propres et, notamment, ici, des performances initiales sur différentes dimensions. Mais il convient de cerner et de sérier, en premier lieu, les observations réalisées.

a) Certaines variables n'apparaissent dans aucune des structures considérées, mais certaines variables apparaissent dans certaines structures de sous-groupes mais pas dans d'autres.

Aux seuils communs choisis pour le groupe complet et les différents sous groupes (.90, $.85, .80, .75)$, la variable ED n'apparaît dans aucune des structures. Les variables «Signes», «Chiffres» n'apparaissent pas dans matrices + (MAT+), mais apparaissent dans matrices -!(MAT-) ; par contre, les variables M5, M2, FR2, FR3, «Mots» n'apparaissent pas dans matrices - (MAT-) mais apparaissent dans matrices + (MAT+).

Pour illustrer encore une fois les considérations ci-dessus, examinons concrètement ce que cela peut signifier cette proposition!: «M5, M2, FR2, FR3, «Mots» n'apparaissent pas dans matrices - (MAT-)

\footnotetext{
${ }^{66} \mathrm{Au}$ sens de la statistique classique en sciences humaines, à savoir en référence à la position du score du sujet dans le groupe à chaque occasion dans le cadre d'un étalonnage des mesures.
} 
mais apparaissent dans matrices + $(M A T+)$ ). Rappelons que l'épreuve MAT ( Matrices »), qui dissocie les deux groupes considérés selon le niveau de performance réalisé (Ceux qui réalisent des performances au delà de la moyenne du groupe et ceux qui réalisent des performances en deçà de la moyenne du groupe), est constituée de séries d'exercices présentés sous forme d'un carré à quatre cases. Les deux premières cases contiennent chacune un dessin (par exemple!: un rond blanc et un rond noir ou un losange avec un point et un losange sans point). La première des deux cases suivantes, placées sous les deux premières, contient un troisième dessin (par exemple!: un carré blanc ou un carré avec un point). Le but de l'exercice consiste à trouver, pour la quatrième case, un dessin qui présente les mêmes rapports au troisième dessin que le $2^{\text {nd }}$ dessin fait valoir avec le premier (Il résulte de cette règle que les réponses aux exemples simples proposés sont un carré noir ou un carré sans point). Pour les élèves qui ont des performances élevées à cette dernière épreuve (MAT+) à la différence de ceux qui ont de faibles performances (MAT-), les variables M5, M2, FR2, FR3 et «Mots» entreraient dans la composition d'une structure implicative au sens du modèle sousjacent au logiciel CHIC.

M5 correspond à la dimension «réception, traitement, production » de l'évaluation CM2/6 en mathématiques, c'est-à-dire à des aspects renvoyant au traitement synthétique et aux conduites supérieures associées au raisonnement, comme la compréhension d'un énoncé, la lecture, la justification d'une réponse, la comparaison d'aires, l'estimation de mesures, le sens donné aux résultats obtenus, à des problèmes de rangement, des énoncés à complétés, etc. M2 correspond à la dimension "techniques opératoires » de l'évaluation $\mathrm{CM} 2 / 6^{\mathrm{e}}$ en mathématiques, à savoir!: effectuer des opérations sur les entiers, les décimaux, maîtriser la division euclidienne. F2, soit «compréhension 2 », renvoie, dans le cadre de l'évaluation $\mathrm{CM} 2 / 6^{\mathrm{e}}$ en français, aux capacités à construire des informations, F3, soit «expression 1 », renvoie à savoir raconter. La dimension «Mots » relève de la partie de l'épreuve de «!Mémoire à Court Terme!» (MCT) portant sur des mots courants de la langue française. Rappelons que dans cette épreuve les élèves doivent se souvenir des contenus de 12 vignettes présentées pendant $2 \mathrm{mn}$ suivies d'un temps de non visionnement de ces vignettes de $2 \mathrm{mn}$.

Quelle hypothèse interprétative peut-on formuler dans le cas de figure présenté!? L'épreuve «Matrice » traduit, d'une certaine manière, la capacité, dans le cadre du raisonnement analogique, à maîtrise des aspects de compréhension appliqués au codage décodage de figures et à y appliquer des règles, un raisonnement, faisant valoir l'analogie.

L'hypothèse que confirmerait l'analyse des données peut être formulée de la manière suivante. La capacité à bien maîtrisée sur données figurées le raisonnement analogique (épreuve «Matrices ») interviendrait, comme élément «!structurateur!»! du développement dynamique et efficace de dimensions particulières relevant des acquis scolaires. Ces dimensions renvoient, notamment, à l'utilisation du code verbal et du code symbolique formalisé au plan des mathématiques, aux capacités!: à utiliser ces codes afin de traiter du sens et du raisonnement, à effectuer des opérations (M5, M2). Elles renvoient à l'expression de la Mémoire à Court Terme (MCT) portant sur des mots de la langue française («!Mots!»), à la structuration de la maîtrise de la langue et de sa compréhension appliquées à la production sous différents aspects (FR2, FR3).

Les variables M2, M5, n'apparaissent pas dans vocabulaire + (VOC+) mais apparaissent dans vocabulaire - (VOC-). Les variables «Signes», «Chiffres» n'apparaissent pas dans vocabulaire - (VOC-), mais dans vocabulaire + (VOC+). Les variables M2, M5, MAT, «Mots», «Images» n'apparaissent pas dans vocabulaire + $(\mathrm{VOC}+)$ mais apparaissent dans matrices + ! (MAT+). Les variables «Signes», «Chiffres» n'apparaissent pas dans matrices + $(\mathrm{MAT}+)$ mais apparaissent dans vocabulaire + (VOC+), etc.

b) Les liaisons implicatives ne sont pas toujours les mêmes pour les différentes sous populations considérées. Sont-elles toutes incluses dans l'ensemble de liaisons, au même seuil, constatées dans la population totale!? 
Effectivement les liaisons implicatives observées dans les différentes sous populations sont différentes d'une population à une autre mais ne se contredisent pas. Par exemple PRO 1 et PRO 2 peuvent impliquer, mais pas systématiquement aux seuils considérés, DIF 3, et PRO 3 impliquer DIF 3, mais jamais n'apparaît l'inverse. Ceci suggère-t-il que suivant les performances initiales montrant une aisance dans un domaine cognitif donné, différent d'un groupe d'élèves à un autre, les cheminements implicatifs de structuration des habiletés cognitives sont différents, mais passent par des cheminements attestés par le relevé de structures implicatives sans qu'il y ait contradiction entre les structures observées dans la population d'ensemble et les structures observées dans les différentes sous populations!?

Par contre la réponse à la question posée est non. Sous une forme directe ou indirecte les observations des liaisons implicatives recueillies pour au moins une des sous populations considérées ne sont pas toutes incluses (de manière directe ou indirecte) dans celles constatées pour la population dans sa globalité. Pour autant il n'y a pas, là aussi, différentes chaînes implicatives en positions contradictoires les unes par rapport aux autres. Par ailleurs, nous avons déjà relevé que la comparaison entre la population totale et les sous-populations fait apparaître des liaisons pour la population globale n'apparaissant pas dans les Graphes correspondant aux sous-groupes (cf. point a), p. 9).

c) Aux mêmes seuils, les liaisons implicatives observées sont plus ou moins nombreuses en fonction des sous-populations considérées

Pour les sous-populations MAT+ et MAT-, ces liaisons implicatives, aux mêmes seuils choisis, sont respectivement de 41 et 27 . Il y a donc une différence de structure avec un réseau plus dense pour la sous-population la plus performante. Par contre, pour les souspopulations VOC+ et VOC-, la position est inverse. Ces liaisons implicatives sont au nombre de 17 et de 41 . Il y a là des différences considérables, en fonction des épreuves servant de critères à la sélection des sous-populations prises en compte aux mêmes seuils choisis $(.90, .85, .80, .75)$. Tout se passe comme si la structure même des relations entre variables dépendait des niveaux de performances, au moins sur certaines épreuves.

Cet ensemble de remarques met en lumière toute la complexité de l'expression, en fonction des groupes de sujets sélectionnés selon différents critères, de structures sousjacentes aux observations de structures très globales qui peuvent être faites. Pour conclure sur ce point, il est tout à fait vraisemblable que les variables choisies, pour différencier les sous-populations à performances élevées ou faibles sur les contenus considérés, entrent en interaction avec les différentes autres variables, pour elles-mêmes, ou dans le cadre des domaines qu'elles représentent. C'est, sans doute, en ce sens qu'il convient d'interpréter le fait que les variables de mémoire à court terme n'apparaissent pas du tout pour la souspopulation aux performances faibles à l'épreuve vocabulaire, alors que les variables MCT «Chiffres» et «Signes» apparaissent lorsque les performances à cette épreuve vocabulaire sont plus élevées. De même, les variables M2 et M5 n'apparaissent pas lorsque les performances à l'épreuve matrices sont faibles alors qu'elles apparaissent lorsque ces performances sont élevées. Cette interprétation est confirmée lorsqu'on détermine la contribution des variables considérées (MAT et VOC) aux différents chemins implicatifs concernant les autres variables. Tantôt, et à des seuils de valeur significative, c'est la variable vocabulaire qui contribue essentiellement aux «!chemins implicatifs!» ${ }^{67}$ entre d'autres variables, tantôt c'est la variable matrices. Pour d'autres «!chemins implicatifs!», les deux variables semblent contribuer conjointement ${ }^{68}$.

\footnotetext{
${ }^{67}$ Structures en chaîne, de variable.

${ }^{68} \mathrm{Cf}$. article actuellement en préparation.
} 


\subsubsection{Que signifient les regroupements implicatifs de certaines variables} indépendamment, partiellement ou non, d'autres variables considérées?

Les regroupements particuliers portent essentiellement sur les performances, sur supports variés, en rapport avec la mémoire à court terme. Ce domaine traite de capacités en deçà de la structuration cognitive actualisée à cette étape du développement correspondant à l'âge considéré. Les capacités, ici mesurées, renvoient, sous le mode implicatif, au même niveau limité de relations avec les autres variables retenues que celui déjà repéré au plan des corrélations et des différentes $\mathrm{ACP}^{69}$ réalisées (cf. [C.!Pellois, 2003(a)]).

Pour les autres domaines, acquis scolaires et performances cognitives, les intrications implicatives entre les variables qui les caractérisent sont multiples au plan de la population traitée globalement. Ces intrications soulignent les relations complexes qu'entretiennent les variables relevant de ces deux domaines.

Vraisemblablement, les relations complexes observées méritent une analyse plus fine. Celle-ci, rapidement esquissée ci-dessous, donnera lieu, par ailleurs, à une publication particulière. Le poids de certaines performances cognitives, comme celles référent à l'analogie verbale (AV), en rapport, ici, avec des capacités cognitives particulières, paraît être important et sous tendre d'autres performances et surtout des acquis scolaires (savoirs et savoir faire) particulièrement centraux (mais pas tous), comme, par exemple : FR1 (compréhension de texte), FR3 (expression), M4 (figures géométriques), M1 (numération et nombres décimaux), FR4 (connaissance du code), M5 (réception, traitement, production). Le sens des opérations (M3) semble, lui, dépendre davantage d'autres performances (DIF!: recherche de différences!; A2C!: logique de classes, ici, en particulier, intersection de classes, ou SN (selon les occasions)!: relations sur données numériques).

\section{CONCLUSION PROVISOIRE...}

5.1. LES DIFFICULTÉS À FONDER DANS LE DOMAINE DES SCIENCES HUMAINES, L'INTÉRET ET LA PERTINENCE DE L'ANALYSE IMPLICATIVE!: UNE QUESTION DE CONTEXTE THÉORIQUE ET MÉTHODOLOGIQUE!?

Le logiciel CHIC, notamment sous l'angle de l'analyse implicative évoquée ici, est un outil puissant de traitement de données. Sa pertinence n'est nullement remise en question mais ce logiciel oblige, comme tout outil statistique, à prendre toutes les précautions méthodologiques nécessaires. En particulier, le contexte interprétatif des données est fondamental. Le sens qui peut être donné aux résultats obtenus nécessite beaucoup de prudence.

L'utilisation qui peut en être faite, comme, d'ailleurs, pour d'autres outils d'analyses structurales de données, sur un mode exploratoire reste largement problématique. L'utilisation sur un mode confirmatoire est la plus pertinente, ceci afin d'échapper aux multiples artefacts induits lorsque ne sont pas maîtrisés, par des grilles de lecture adaptées, les différentes interprétations possibles des résultats obtenus. Cette utilisation sur un mode confirmatoire est d'autant plus valide que l'arrière plan théorique est largement structuré et que les hypothèses émises sont des hypothèses fortes. C'est-àdire des hypothèses pour lesquelles le risque pris, d'infirmation de l'hypothèse, est élevé.

\footnotetext{
${ }^{69}$ Analyses en Composantes Principales.
} 
La confusion entre les deux formes de précédence, «temporelle » et au sens de la relation «implicative » est à éviter, sauf intentions particulières et à condition de traiter de la précédence «temporelle » entre différents types de variables, selon des méthodologies adaptées $^{70}$, des modèles théoriquement structurés et situés au regard de la littérature sur ces questions, et selon des hypothèses émises clairement spécifiées. La précédence «temporelle » à visée «implicative » entre performances portant, en particulier, sur la même variable n'a pas de sens, en particulier dans le cas de la progression des performances dans le temps, pour les sujets considérés.

Par ailleurs, le statut des variables, leur signification pragmatique ou théorique, leurs rapports entre elles, les évolutions différentielles, notamment intra-individuelles ${ }^{71}$, de performances, tout ceci est à examiner de très près avant qu'une analyse en termes d'inférences causales ne puisse être développée.

Enfin, en situation de recherche de terrain, comme en laboratoire, mais de manière plus réduite, et donc dans une situation moins «!risquée!» au plan des interprétations, l'influence de variables non maîtrisées, dans le cadre de l'analyse interprétative, peuvent avoir des effets susceptibles d'interférer avec ceux liés, par hypothèse, aux dimensions considérées. Le chercheur n'est pas supposé maîtriser systématiquement l'ensemble des variables des contextes considérés ce qui est susceptible de rendre encore plus hypothétiques les interprétations qu'il donne aux résultats obtenus.

Les possibilités de traitement de données qu'offre le logiciel CHIC, notamment sous l'angle de l'analyse implicative, constituent, cependant, une avancée notable dans la conception et la construction d'outils statistiques susceptibles de traiter des données sous l'angle structural. Dans ce cadre de l'analyse structurale de données, il constitue un progrès au regard, par exemple, du logiciel LISREL qui ne présente pas cette même possibilité de traiter de l'imputation causale sous l'angle de l'analyse implicative.

\section{2. À QUELS RÉSULTATS, ICI RAPIDEMENT ÉVOQUÉS, ABOUTIT LA RECHERCHE À PROPOS DE LAQUELLE CETTE RÉFLEXION MÉTHODOLOGIQUE A ÉTÉ DÉVELOPPÉE!?}

$\mathrm{Au} v u$ des différentes remarques et réserves méthodologiques, de l'analyse critique développée, il ne paraît pas évident de faire valoir des résultats qui auraient un sens autre que les conclusions triviales auxquels des développements ci-dessus ont pu aboutir. Pourtant quelques éléments peuvent être retenus et seront développés par ailleurs ${ }^{72}$.

Tout d'abord on peut constater, à propos des résultats, pour chaque occasion, que les mesures concernant les capacités cognitives, à cet âge, pour une partie d'entre elles, «!précèdent!», dans le second sens évoqué plus haut, les mesures concernant les acquis scolaires, et pour une autres partie, sont précédées par les mesures concernant des capacités cognitives différentes. Ce qui tendrait à dire que les deux dimensions alimentent,

\footnotetext{
${ }^{70}$ Comme cela a été précédemment évoqué, par exemple, une performance à un niveau donné sur une dimension $X$, à l'occasion 1 de la mesure, «!précède!» l'accroissement de performances sur une dimension $Y$ de l'occasion 1 à l'occasion 2 de la mesure. Cette observation peut avoir du sens si celle-ci fait valoir, notamment, qu'il y a absence d'observation de cet accroissement en l'absence de performance au niveau donné sur la dimension $X$, à l'occasion 1 de la mesure, elle-même associée à l'observation qu'aucune absence d'accroissement n'apparaît pour $Y$ si la performance à un niveau donné sur une dimension $X$ existe à l'occasion 1 de la mesure (Ici il s'agirait de la position corrélative attestant d'une implication stricte).

${ }^{71}$ Voire les fluctuations des scores, donc la plus ou moins grande fidélité (au sens psychométrique du terme) des mesures réalisées à partir des épreuves considérées.

${ }^{72} \mathrm{Cf}$. article en préparation, déjà évoqué.
} 
l'une et l'autre, le développement du sujet. Les capacités mesurées à propos du langage, au plan cognitif, dépendent, semble-t-il, de la bonne maîtrise d'acquis scolaires et de capacités cognitives sur d'autres dimensions.

Ensuite les différentes structures apparaissant pour différentes sous populations suggèreraient des parcours de structuration à la fois cognitive et d'acquisition de connaissances scolaires, différents pour les différents groupes d'élèves considérés, en fonction de leurs performances initiales, en particulier dans deux capacités cognitives différentes.

Des performances cognitives particulières en précèdent d'autres, tel est le cas de l'épreuve analogies verbales. Ce qui suggère que l'élaboration cognitive, pour le sujet, la maîtrise efficace de contenus scolaires implique une capacité à développer de manière pertinente le raisonnement analogique ${ }^{73}$ sous ses aspects verbaux, notamment.

Enfin l'étude portant sur les performances cognitives élevées réalisées sur des épreuves singulières $^{74}$ semblent donner des résultats contradictoires ${ }^{75}$ au regard de l'hypothèse qui consisterait à les considérer comme particulièrement associées à une capacité importante d'organisation implicative (cf. Graphes 5,6,7,8), tout à la fois des autres habiletés cognitives et des acquis scolaires. Il serait intéressant d'analyser, dans le cadre de la psychologie différentielle du développement, ce que sous-tendent ces différentes structurations multiples, portant sur des regroupements de variables, à la fois communes et différentes les unes des autres. Malgré de nombreux travaux portant sur le développement cognitif, variable en fonction des groupes et des âges considérés, la question posée par cette expression différentielle reste encore très largement à explorer.

\footnotetext{
${ }^{73}$ Ceci renvoie à des résultats dont rend compte la littérature dans le domaine des apprentissages (cf. [J. F. Richard, 1990]).

${ }^{74}$ Épreuves « matrices » et «vocabulaire » de l'ECNI.

${ }^{75} \mathrm{Cf}$. notamment, point 4.2.4., c).
} 


\section{BIBLIOGRAPHIE}

BACHER F., Les enquêtes en psychologie, tome 1!: Description, Lille, Presses Universitaires de Lille, coll. Sciences humaines, Université de Lille II, 19835(a).

BACHER F., Les enquêtes en psychologie, tome 2 : Explication, Lille, Presses Universitaires de Lille, coll. Sciences humaines, Université de Lille II, 1983(b).

BACHER F., «!Les modèles structuraux en psychologie, présentation d'un modèle!: LISREL ( $1^{\text {ère }}$ partie)!», Le travail humain, tome 50, 4, 1987, p. 347-370.

BACHER F., «!Les modèles structuraux en psychologie, présentation d'un modèle!: LISREL $\left(2^{\mathrm{e}}\right.$ partie)!», Le travail humain, tome 51, 4, 1988, p. 273-288.

BACHER F., «!L'utilisation des modèles dans l'analyse des structures de covariance!», L'année psychologique 99, 1999, p. 99-122.

BAILLEUL M., «!L'analyse statistique implicative!: variables modales et contributions des sujets!», Actes du colloque!: Méthodes d'analyses statistiques multidimentionnelles en didactique des mathématiques, Caen, 1995, p. 145-153.

BAILLEUL M., «!Les réseaux implicatifs pour mettre en évidence des représentations!», Mathématiques et Sciences humaines 154-155, 2001, p. 31-46.

HUTEAU M., LAUTREY J., Évaluer l'intelligence, psychométrie cognitive, Paris, Presses Universitaires de France, Coll. Psychologie sciences de la pensée, 1999.

GRAS R., «!L'analyse statistique implicative. Introduction!», Actes du colloque : Méthodes d'analyses statistiques multidimentionnelles en didactique des mathématiques, Caen, 1995, p. 129-143.

GRAS R. et coll., L'implication statistique, nouvelle méthode exploratoire de données, La pensée sauvage, coll. Recherches en didactique des mathématiques, 1996.

GRAS R., «!Panorama du développement de l'A.S.I. à partir de situations fondatrices!», Actes des Troisièmes rencontres internationales ASI, Palerme, 2005, p. 9-33.

HOC J. M., L'analyse planifiée des données, Paris, Presses Universitaires de France, coll. Le psychologue, 1983.

INHElder B., PIAGet, J., De la logique de l'enfant à la logique de l'adolescent, Paris, Presses Universitaires de France, coll. Bibliothèque philosophique contemporaine, 1970, [1 ${ }^{\text {ère }}$ édition!: 1959].

JUHEL J., «!Les systèmes dynamiques non linéaires dans l'étude de la variabilité cognitive!», Actes du Colloque International : La complexité, ses formes, ses traitements, ses effets, Cahiers de la MRSH, n’!spécial, Université de Caen Basse-Normandie, avril 2005, p. 193-216.

LAUTREY J. (sous la direction de), Universel et différentiel en psychologie, Paris, Presses Universitaires de France, coll. Psychologie d'aujourd'hui, 1995.

PELLOIS C., «!Apprentissages et développement de la personne dans l'enseignement et la formation!», tome 1!: Du rationnel au complexe, Paris, l'Harmattan, coll. Recherches et innovations, 2002.

PELLOIS C., «!Enseignement et formation!: le développement de la personne!», tome 2!: Le complexe, Paris, l'Harmattan, coll. Recherches et innovations, 2003(a).

PELLOIS C., «!Effets observés à propos d'ateliers d'aide au développement cognitif en collège!», Psychologie différentielle : recherches et réflexions, (sous la direction de A. Vom Hofe, H. Charvin, J.-L. Bernaud, D. Guédon), Actes des $15^{\mathrm{e}}$ journées de psychologies différentielles L'étude des différences individuelles, Université de Rouen, Rennes, Presses Universitaires de Rennes, 2003(b).

PIAGET J., La psychologie de l'intelligence, Paris, Armand Colin, coll. Prisme, 1967.

PIAGET J., La prise de conscience, Paris, Presses Universitaires de France, coll. Psychologie d'aujourd'hui, 1974(a). 
PIAGET J., Réussir et comprendre, Paris, Presses Universitaires de France, coll. Psychologie d'aujourd'hui, 1974(b).

PIAGET J., L'équilibration des structures cognitives problème central du développement, Paris, Presses Universitaires de France, coll. Études d'épistémologie génétique, tome XXXIII, 1975.

PIAGET J., La naissance de l'intelligence chez l'enfant, Delachaux et Niestle, $9^{\mathrm{e}}$ éd., 1977.

RICHARD J.-F., Les activités mentales, comprendre, raisonner, trouver des solutions, Paris, Armand Colin, 1990.

REUCHLIN M., Totalités, éléments, structures en psychologie, Paris, Presses Universitaire de France, 1995. 